\title{
ABO-Incompatible Adult Living Donor Liver Transplantation in the Era of Rituximab: A Systematic Review and Meta-Analysis
}

\author{
Dipesh Kumar Yadav ${ }^{D},{ }^{1}$ Yong Fei Hua, ${ }^{1,2}$ Xueli Bai, ${ }^{1}$ Jianying Lou, ${ }^{3}$ Risheng Que, \\ Shunling Gao, ${ }^{1}$ Yun Zhang, ${ }^{3}$ Ji Wang, ${ }^{1}$ Qinfen Xie, ${ }^{4}$ Muhammad Ibrahim Alhadi Edoo, ${ }^{1}$ \\ Vikram Kumar Chutturghoon, ${ }^{1}$ and Tingbo Liang $\mathbb{D}^{1}$ \\ ${ }^{1}$ Department of Hepatobiliary and Pancreatic Surgery, The First Affiliated Hospital, Zhejiang University School of Medicine, \\ Hangzhou, 310003 Zhejiang, China \\ ${ }^{2}$ Department of Hepatobiliary and Pancreatic Surgery, Ningbo Medical Center Lihuili Eastern Hospital, Medical School of \\ Ningbo University, Ningbo, 315041 Zhejiang, China \\ ${ }^{3}$ Department of Hepatobiliary and Pancreatic Surgery, The Second Affiliated Hospital, Zhejiang University School of Medicine, \\ 88 Jiefang Road, Hangzhou, 310009 Zhejiang, China \\ ${ }^{4}$ Department of Hepatobiliary Surgery, Shulan (Hangzhou) Hospital, Hangzhou, 310000 Zhejiang, China
}

Correspondence should be addressed to Tingbo Liang; liangtingbo@zju.edu.cn

Received 17 February 2019; Accepted 7 May 2019; Published 11 June 2019

Academic Editor: Cataldo Doria

Copyright (C) 2019 Dipesh Kumar Yadav et al. This is an open access article distributed under the Creative Commons Attribution License, which permits unrestricted use, distribution, and reproduction in any medium, provided the original work is properly cited.

\begin{abstract}
Aim. The primary aim of this study is to compare the short- and long-term outcomes between ABO-incompatible (ABOi) adult living donor liver transplantation (ALDLT) with rituximab prophylaxis and ABO-compatible (ABOc) ALDLT. Background. The strategy of ABOi liver transplantation (LT) was originated initially to increase the donor pool and to enable liver transplantation in emergency conditions. However, ABOi ALDLT remains a controversial approach in comparison to ABOc ALDLT. Methods. PubMed, Embase, and the Cochrane Library study search were accomplished to recognize studies comparing ABOi and ABOc ALDLT. Meta-analyses were conducted based on the evaluation of heterogeneity using a fixed-effect model and a random-effect model to assess the short- and long-term outcomes following ABOi ALDLT with rituximab prophylaxis. Results. Nine studies comprising a total of 3,922 patients $(\mathrm{ABOi}=671$ and $\mathrm{ABOC}=3,251)$ were identified. There was no significant difference between $\mathrm{ABO}$ and $\mathrm{ABOc}$ groups for 1-year, 3-year, and 5-year OS and graft survival, respectively. Moreover, 1-year and 3-year OS and DFS were similar between both groups for HCC patients. However, ABOi ALDLT had higher incidences of CMV infection, AMR, overall biliary complications, and biliary stricture than ABOc ALDLT and had other comparable postoperative complications. Conclusion. Our meta-analysis included studies comparing ABOi and ABOc ALDLT after the introduction of rituximab in a desensitization protocol for ABOi ALDLT. The results of ABOi ALDLT were comparable with those of ABOc ALDLT. However, biliary complications, CMV infection, and AMR remain a concern in the era of rituximab.
\end{abstract}

\section{Introduction}

Liver transplantation (LT) has now become an ideal treatment option for patients with liver cancer and end-stage liver diseases $[1,2]$; however, its use is restricted due to a limited donor pool $[3,4]$. In past decades, different attempts and breakthroughs have been made to increase the donor pool [5]. One of them is living donor liver transplantation (LDLT); this applies both for urgent and elective LT $[5,6]$.
Moreover, in the shortage of $\mathrm{ABO}$-compatible (ABOc) donors and to increase the donor pool, $\mathrm{ABO}$-incompatible (ABOi) LT remains the only option for many with a rapidly worsening liver function or for one who remains on a long waiting list $[7,8]$. The liver is considered as an immuneprivileged organ since it has a low incidence of humoral rejection unlike the kidney and the heart $[9,10]$. Taking this into consideration, different innovative $B$ cell desensitization protocols, such as the use of total plasma exchange (TPE), 
double-filtration plasmapheresis, local graft infusion therapy (LGIT), splenectomy, rituximab, mycophenolate mofetil (MMF), and intravenous immunoglobulin G (IVIG), have been used to breach the blood group barrier leading to significant advancements in the outcome of ABOi [11, 12]. Thus, $\mathrm{ABO}$ is no longer contemplated as a contraindication for LT.

ABOi LDLT pediatric patients are considered safe and with acceptable results probably because of their immature immune system $[11,13,14]$. However, the safety of $\mathrm{ABO}$ adult LDLT (ALDLT) is debatable among the transplant community due to different risks associated to it, especially earlier graft loss, acute cellular rejection (ACR), antibodymediated rejection (AMR), and vascular and biliary complications, compared to those associated to ABOc ALDLT [15-17]. Likewise, hepatocellular carcinoma (HCC) recurrence after $\mathrm{ABOc} A L D L T$ remains another major concern due to the patient's immunosuppressed state [18]. An effective desensitization protocol for ABOi ALDLT is very demanding. The introduction of rituximab, an anti-CD20 monoclonal antibody, to the desensitization protocol has brought about a significant reduction in the incidence of AMR and has improved the outcome of ABOi ALDLT [12, $19,20]$. Rituximab acts on the CD20 antigen present on B cells, thus reducing the production of $\mathrm{B}$ cells which are mainly responsible for acute rejection and AMR [21, 22]. Monteiro et al. [23] were the first to report the case of rituximab use in ABOi LT in 2003. Since then, there have been several studies that have reported on rituximab prophylaxis in ABOi ALDLT [22, 24-31].

To our knowledge, no systematic evaluations have been performed to determine the effectiveness and safety of the rituximab regimen in $\mathrm{ABO}$ ALDLT. This study is aimed at comparing the short- and long-term outcomes between ABOi ALDLT with rituximab prophylaxis and ABOc ALDLT. Additionally, this meta-analysis also intended to assess the long-term outcomes of HCC patients following ABOi ALDLT with rituximab prophylaxis compared to those of HCC patients following ABOc ALDLT.

\section{Methods}

2.1. Search Strategy. Qualified studies for this systematic review and meta-analyses were selected following the earlier settled convention with the PubMed/MEDLINE, Embase, and Cochrane Library databases by two authors (DY and YFH), using a combination of the following Medical Subject Headings (MeSH) and non-MeSH terms: liver transplantation, ABO-incompatible liver transplantation, ABOcompatible liver transplantation, hepatocellular carcinoma, tumor recurrence, primary liver carcinoma, and HCC. Additionally, the pertinent bibliography lists of articles were taken into consideration to distinguish other important studies. After preliminary screening, duplicate articles, abstracts, or unpublished studies were ruled out. The Preferred Reporting Items for Systematic Reviews and Meta-Analysis (PRISMA) guidelines were used to perform this meta-analysis [32].

2.2. Study Selection. We considered both retrospective and prospective studies eligible for this meta-analysis with respect to the outcomes. Additionally, considering the outcome goals and ensuring the quality of this meta-analysis, we only considered fully published studies and excluded studies with only abstracts. Additionally, we designed the following predefined eligibility criteria for the selection of studies with at least one outcome of interest.

\subsubsection{Inclusion Criteria}

(1) The study should have a definition of ABOi ALDLT and ABOc ALDLT. ABOi ALDLT includes the following donor-to-recipient combinations: $\mathrm{A}$ to $\mathrm{B}$ and $\mathrm{O}$; $\mathrm{B}$ to $\mathrm{A}$ and $\mathrm{O}$; and $\mathrm{AB}$ to $\mathrm{A}, \mathrm{B}$, and $\mathrm{O}$. Other combinations are regarded as $\mathrm{ABOc}$, including the $\mathrm{ABO}$ identical blood group

(2) The study should contain ALDLT and should compare short- and long-term results between ABOi ALDLT and ABOc ALDLT

(3) The study should have sufficient data to conduct a meta-analysis

(4) Adult participants ( $>16$ years of age).

\subsubsection{Exclusion Criteria}

(1) A study without human subjects

(2) A study with pediatric patients and deceased donor liver transplant

(3) A study containing advanced disease stage or extrahepatic metastases

(4) A study with no comparison between ABOi ALDLT and ABOc ALDLT

(5) A study with a multiorgan transplant

(6) A study with older patients above 70 years

(7) A study with duplicate data from the same institution

(8) Publications such as review articles, editorials, case reports, conferences, and letters

2.3. Data Extraction. All data were extracted according to the study selection criteria and were abstracted in a systematized data abstraction form using Microsoft Excel 2007 (Microsoft Corp.). The extracted data included the first author, study characteristics (publication year, country, and study design), participant characteristics (average age of the recipients, sample size of ABOi and ABOc ALDLT, pretransplant MELD score, disease characteristics, pretransplant AFP level for HCC patients, number and size of tumors for HCC patients, pretransplant therapies, hospital stay, and the duration of follow-up), and outcomes (biliary complications, infectious complications, vascular complications, acute cellular rejection, antibody-mediated rejection (AMR), graft survival, overall survival (OS), and disease-free survival (DFS) for HCC patients). Moreover, in case of insufficient data, investigators were approached to collect more relevant 


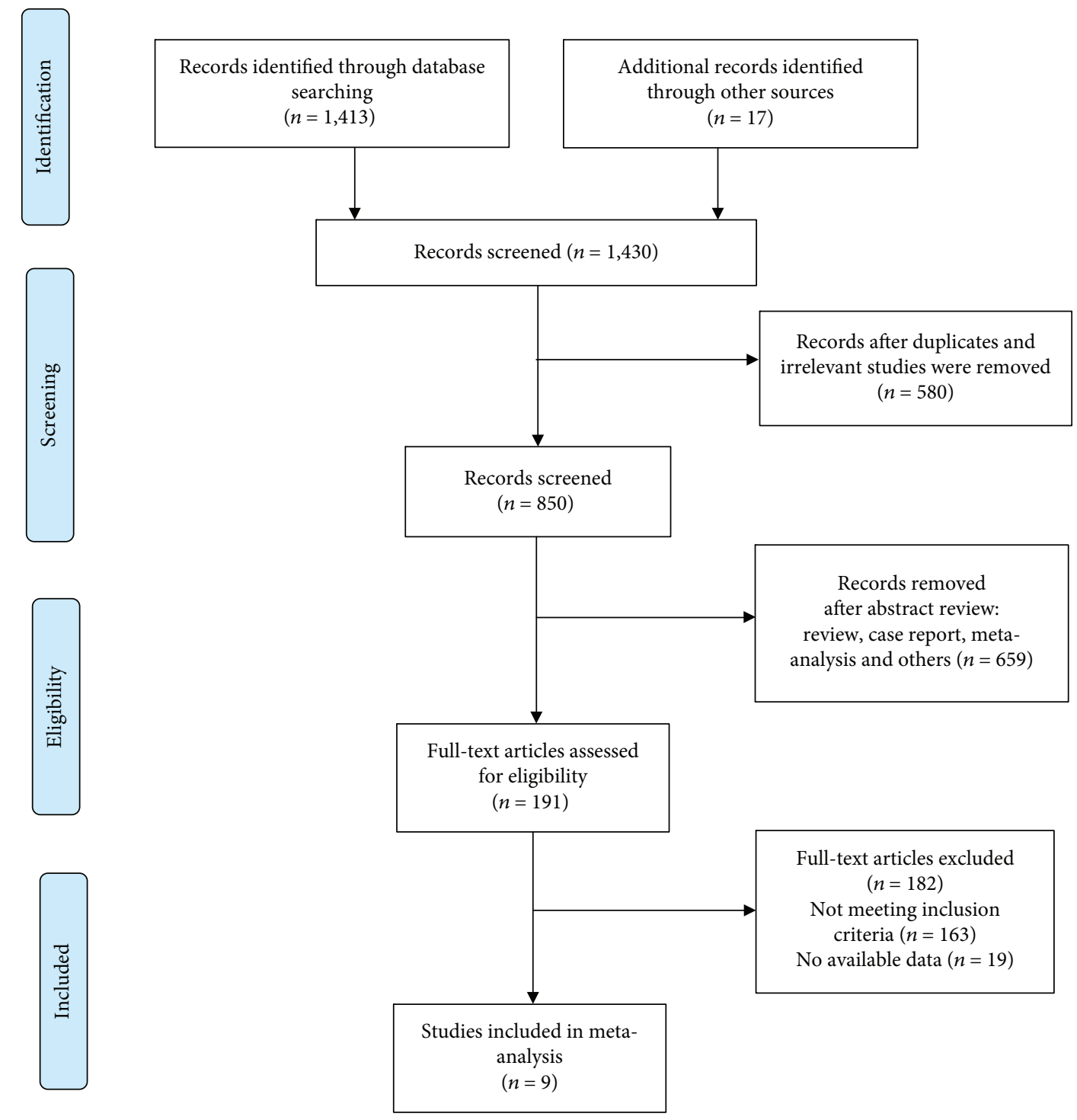

Figure 1: Preferred reporting items for systematic review and meta-analysis study flow diagram for literature search.

results. Conflicts in data extraction were resolved by discussion or consensus with a 3 rd reviewer.

2.4. Quality Assessment. The quality of included studies was evaluated with the Newcastle-Ottawa scale (NOS) [33]. The scale comprises 3 assessment factors: (1) assessment of a selection of the study groups; (2) comparability of the 2 groups; and (3) outcome assessment. The NOS ranges from 0 to 9 . Studies with scores of 7 points and above were considered to be of high quality, those with 4-6 points were considered to be of moderate quality, and those with less than 4 points were considered to be of lower quality (Supplementary Table 1).

2.5. Statistical Analysis. All results are accounted for as in the original articles and were double-checked. A meta-analysis was carried out with RevMan Version 5.3 (Review Manager, Copenhagen: The Nordic Cochrane Center, The Cochrane Collaboration, 2014). Outcomes are calculated as pooled odds ratios (ORs) and standard mean difference (SMD) with corresponding 95\% confidence intervals (CIs). Fixed-effect or random-effect models were utilized to compute summary estimates based on the evaluation of heterogeneity. Overall effects were evaluated by utilizing the $Z$-test, and heterogeneity was tested by using Cochran's $\chi^{2}$ test. The $I^{2}$ statistic was utilized to evaluate heterogeneity, which was characterized as low, moderate, or high with $I^{2}$ esteemed at $>25 \%,>50 \%$, and $>75 \%$, respectively [34]. Two-sided $P$ values less than 0.05 were considered significant.

\section{Results}

3.1. Study Search and Included Studies. The database scans recognized 1,430 references for assessment (Figure 1), and 191 full-text articles were assessed for eligibility. Furthermore, 182 articles were excluded (articles that did not meet the inclusion criteria $(n=163)$ and those with insufficient data $(n=19))$. The remaining 9 retrospective studies between 
2015 and 2018 were eligible according to the inclusion criteria and were included in this meta-analysis, with a total of 3,922 patients $(A B O i=671$ and $A B O c=3,251)$ (Table 1$)$ [22, 24-31]. Although we identified 9 studies for inclusion in the analysis, two of the studies (study nos. 1 and 2) $[25,26]$ identified were from the same institutions in Korea as those of study nos. 3 and 4 [27, 28]. These two studies were only identified to calculate the outcome of interest for $\mathrm{ABO}$ ALDLT in HCC patients and were not used for other calculations in this meta-analysis.

\section{Meta-Analysis}

\subsection{Primary Outcome}

4.1.1. Patients' Preoperative and Perioperative Outcomes. Meta-analyses of preoperative and perioperative outcomes are shown in Figure 2. To assess the outcome measurement of the MELD score, a total of 2,764 patients were incorporated in 7 studies $[22,24,27-31]$. The $\chi^{2}$ test $(P<0.00001$ and $I^{2}=91 \%$ ) and meta-analysis using a random-effect model revealed that there was no significant difference in the MELD score between the $\mathrm{ABOi}$ and $\mathrm{ABOc}$ groups (SMD: $-1.31,95 \% \mathrm{CI}:-2.83$ to $0.21, P=0.09$, Figure $2(\mathrm{a})$ ).

After classifying the data according to ischemia type, i.e., warm ischemia and cold ischemia, a meta-analysis using a random-effect model revealed that there was no significant difference in warm ischemia time (SMD: 1.14, 95\% CI: -2.61 to $4.89, P=0.55$, Figure $2(\mathrm{~b}))[22,27,28,31]$ between the ABOi and ABOc groups. However, a metaanalysis using a fixed-effect model revealed that cold ischemia time was significantly shorter in the ABOi group than in the ABOc group (SMD: $-3.23,95 \% \mathrm{CI}:-4.62$ to -1.84 , $P<0.00001$, Figure 2(c)) $[22,27,28,31]$.

4.1.2. Postoperative Short-Term Outcomes. Meta-analyses of postoperative short-term outcomes, i.e., infectious complications, vascular complications, hospital stay, and biliary complications, are shown in Figure 3.

(1) Infectious Complications. Under subgroup analysis, overall infections, bacterial infections, fungal infections, and cytomegalovirus (CMV) infections were taken under consideration for meta-analysis.

A meta-analysis using a fixed-effect model revealed that there was no significant difference between the ABOi and $\mathrm{ABOc}$ groups for overall infections (OR: 1.25, 95\% CI: 0.50 to $3.12, P=0.63$, Figure $3(\mathrm{a}))[24,29]$, bacterial infections (OR: $0.69,95 \% \mathrm{CI}: 0.42$ to $1.15, P=0.16$, Figure $3(\mathrm{~b})$ ) $[27,28,31]$, and fungal infections (OR: 0.65, 95\% CI: 0.31 to $1.34, P=0.24$, Figure $3(\mathrm{c}))[27,28]$, respectively. However, a meta-analysis using a fixed-effect model revealed that CMV infection was significantly higher in the ABOi group than in the ABOc group (OR: $1.85,95 \% \mathrm{CI}: 1.13$ to $3.03, P=0.01$, Figure 3(d)) [22, 27, 28, 31].

(2) Vascular Complications. Under subgroup analysis, hepatic artery stenosis, portal vein stenosis, and bleeding were taken under consideration for meta-analysis. A metaanalysis using a fixed-effect model revealed that there was no significant difference between the ABOi and $\mathrm{ABOc}$ groups for hepatic artery stenosis (OR: 2.86, 95\% CI: 0.93 to $8.76, P=0.07$, Figure $3(\mathrm{e}))[22,27,29,31]$, portal vein stenosis (OR: $1.19,95 \% \mathrm{CI}: 0.30$ to $4.65, P=0.80$, Figure 3(f)) [27, 29, 31], and bleeding (OR: 0.88, 95\% CI: 0.49 to $1.59, P=0.67$, Figure $3(\mathrm{~g}))[22,27,29]$, respectively.

(3) Biliary Complications. After classifying data according to biliary complication types, i.e., overall biliary complications, biliary leakage, and biliary stricture, a meta-analysis revealed that there was no significant difference between the ABOi and $\mathrm{ABOc}$ groups for biliary leakage (OR: 1.13, 95\% CI: 0.54 to $2.36, P=0.75$, Figure $3(\mathrm{~h}))[22,28,29]$. However, overall biliary complications (OR: $1.47,95 \% \mathrm{CI}: 1.07$ to 2.03, $P=0.02$, Figure 3(i)) $[24,27,28]$ and biliary stricture (OR: $1.49,95 \%$ CI: 1.14 to $1.96, P=0.004$, Figure 3(j)) [22, 27-31] were significantly higher in the ABOi group than in the $\mathrm{ABOc}$ group.

(4) Hospital Stay. To assess the outcome measurement of hospital stay, a total of 842 patients were incorporated in 5 studies $[22,24,28,29,31]$. The $\chi^{2}$ test $(P=0.12$ and $\left.I^{2}=45 \%\right)$ and meta-analysis using a fixed-effect model revealed that hospital stay was significantly longer in the ABOi group than in the ABOc group (SMD: 3.39, 95\% CI: 2.14 to $4.64, P<0.00001$, Figure $3(\mathrm{k}))$.

4.1.3. Postoperative Long-Term Outcomes. Meta-analyses of postoperative long-term outcomes, i.e., graft rejection, overall survival (OS), and graft survival, are shown in Figure 4.

(1) Graft Rejection. After classifying data according to graft rejection types, i.e., antibody-mediated rejection (AMR) and acute cellular rejection (ACR), a meta-analysis using a random-effect model revealed that $A M R$ was significantly higher in the ABOi group than in the ABOc group (OR: 21.58, 95\% CI: 2.45 to $190.07 .13, P=0.006$, Figure $4(\mathrm{a})$ ) [22, 24, 27-31]. However, a meta-analysis using a fixedeffect model revealed that there was no significant difference in ACR between the ABOi and ABOc groups (OR: $0.98,95 \% \mathrm{CI}: 0.67$ to $1.43, P=0.90$, Figure $4(\mathrm{~b}))[22,24$, $27-31]$.

(2) Overall Survival (OS). To assess the outcome measurement of overall survival, data were classified according to 1year, 3-year, and 5-year OS, respectively. A meta-analysis revealed that there was no significant difference between the $\mathrm{ABO}$ and $\mathrm{ABOc}$ groups for 1-year (OR: 0.88, 95\% CI: 0.59 to $1.30, P=0.51$, Figure $4(\mathrm{c}))[22,24,27,28,30]$, 3-year (OR: $1.02,95 \%$ CI: 0.73 to $1.43, P=0.91$, Figure $4(\mathrm{~d})$ ) [22, 24, 27, 28, 30], and 5-year ( OR: 1.00, 95\% CI: 0.68 to $1.47, P=0.13$, Figure $4(\mathrm{e}))[24,27,30]$ OS, respectively.

(3) Graft Survival. To assess the outcome measurement of graft survival, data were classified according to 1-year, 3-year, and 5-year graft survival, respectively. A meta-analysis revealed that there was no significant difference between 


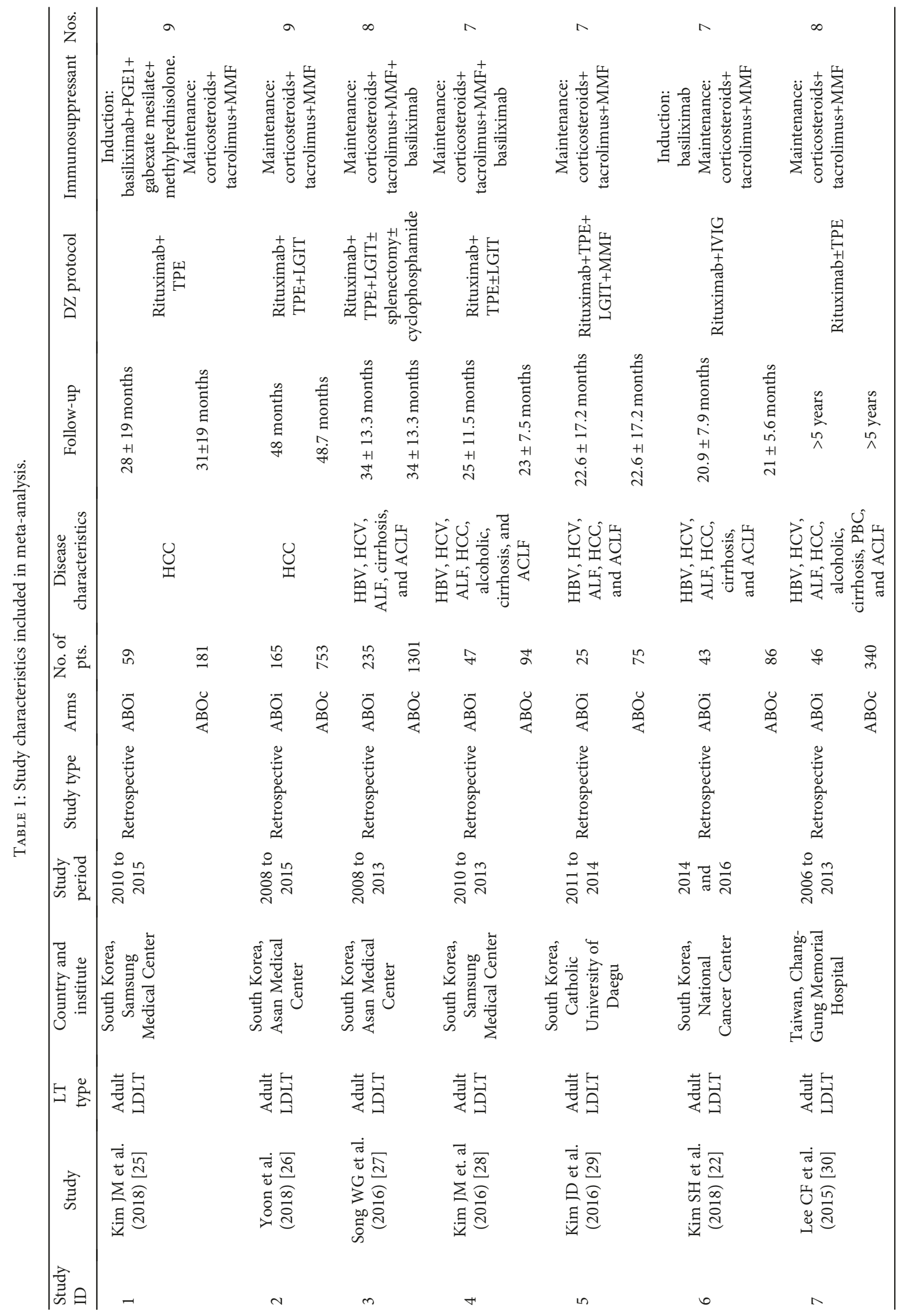




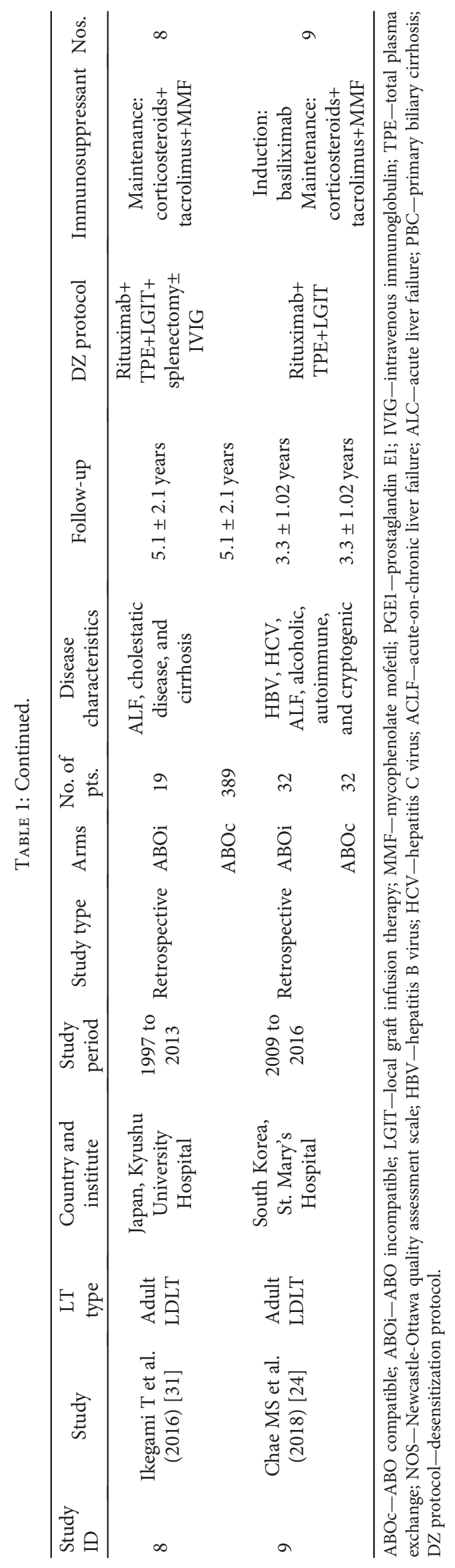




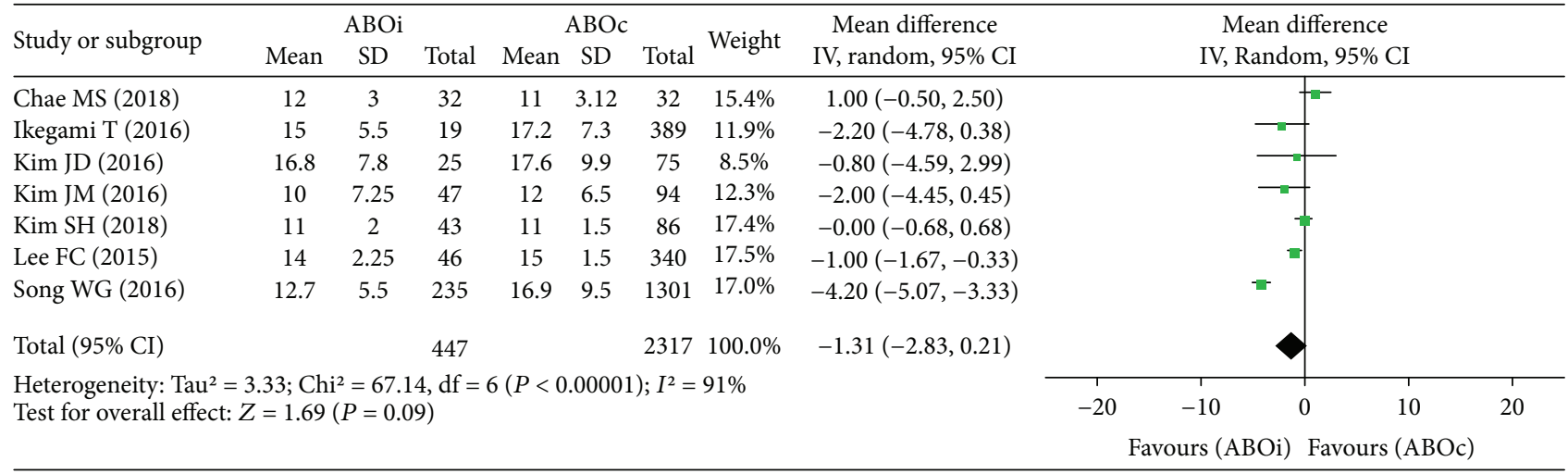

(a) MELD score

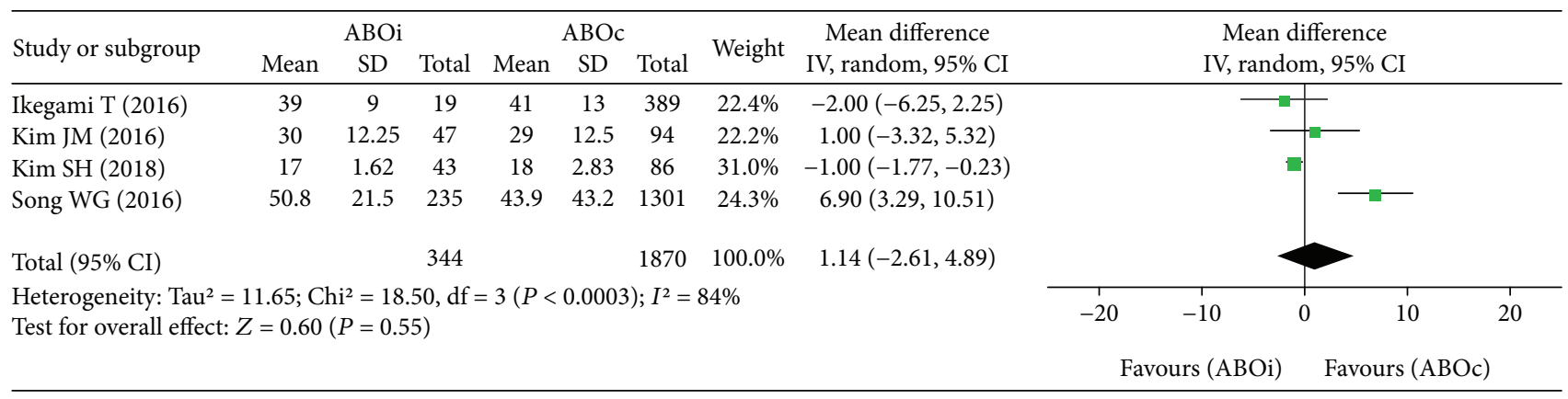

(b) Warm ischemia

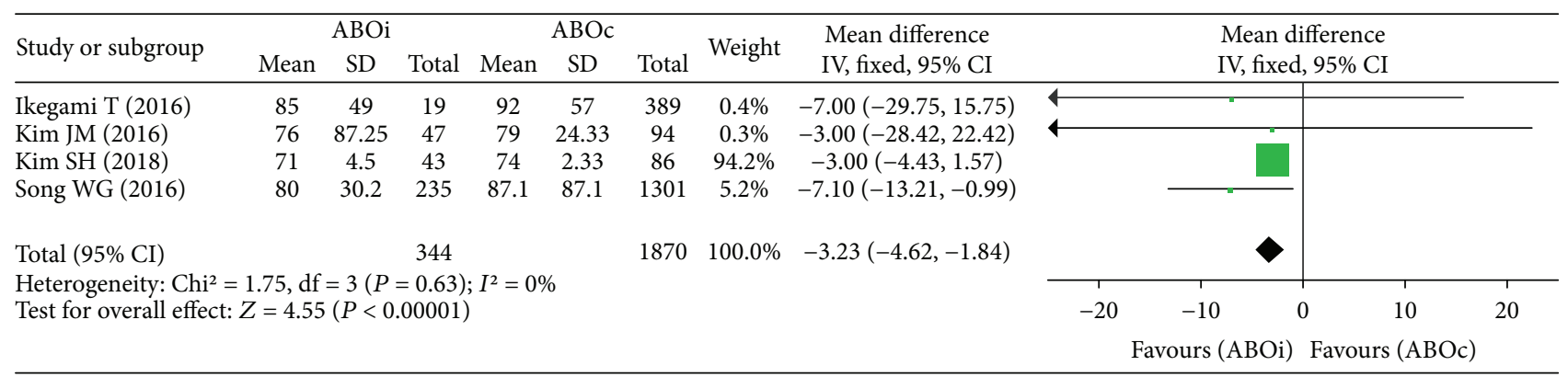

(c) Cold ischemia

FIGURE 2: Forest plot of patients' preoperative and perioperative outcomes: (a) MELD score, (b) warm ischemia, and (c) cold ischemia.

the ABOi and ABOc groups for 1-year (OR: 0.93, 95\% CI: 0.60 to $1.46, P=0.76$, Figure $4(\mathrm{f}))[27,30,31]$, 3-year (OR: $0.84,95 \% \mathrm{CI}: 0.57$ to $1.25, P=0.39$, Figure $4(\mathrm{~g}))[27,30]$, and 5-year (OR: 0.96, 95\% CI: 0.66 to $1.39, P=0.83$, Figure 4(h)) $[27,30,31]$ graft survival, respectively.

4.1.4. Outcome for ABOi ALDLT for Patients with HCC. Meta-analyses of the outcome for ABOi ALDLT for patients with HCC are shown in Supplementary Figure 1. To assess the outcome measurement of ABOi ALDLT for patients with HCC, a total of 1,158 patients were incorporated in 2 studies $[25,26]$. A meta-analysis using a fixed-effect model revealed that there was no significant difference in preoperative AFP level (SMD: $-5.96,95 \%$ CI: -238.26 to 226.34, $P=0.96$, Supplementary Figure 1(a)) between the $\mathrm{ABO}$ and $\mathrm{ABOc}$ groups for patients with HCC. However, the preoperative MELD score was significantly lower in the $\mathrm{ABO}$ group than in the $\mathrm{ABOc}$ group for patients with
HCC (SMD: $-1.13,95 \%$ CI: -1.88 to $-0.38, \quad P=0.003$, Supplementary Figure 1(b)).

A meta-analysis of pretransplant tumor characteristics found that the maximum tumor diameter was significantly smaller in ABOi LDLT than in ABOc ALDLT (SMD: -0.30, 95\% CI: -0.56 to $-0.03, P=0.03$, Supplementary Figure $1(\mathrm{c})$ ). However, the number of tumors was not significantly different among both groups (SMD: -0.22 , 95\% CI: -1.15 to $1.58, P=0.76$, Supplementary Figure $1(\mathrm{~d}))$. There were no useful data for the meta-analysis of tumor size $>3 \mathrm{~cm}$ or tumor nodules more than 3 .

To assess the outcome measurement of overall survival (OS) for HCC patients, data was classified according to 1year and 3-year OS, respectively. There were no useful data to calculate 5-year OS. A meta-analysis revealed that there was no significant difference between the $\mathrm{ABO}$ and $\mathrm{ABOc}$ groups for 1-year (OR: $1.31,95 \% \mathrm{CI}: 0.67$ to $2.56, P=0.43$, Supplementary Figure 1(e)) and 3-year (OR: 1.17, 95\% CI: 


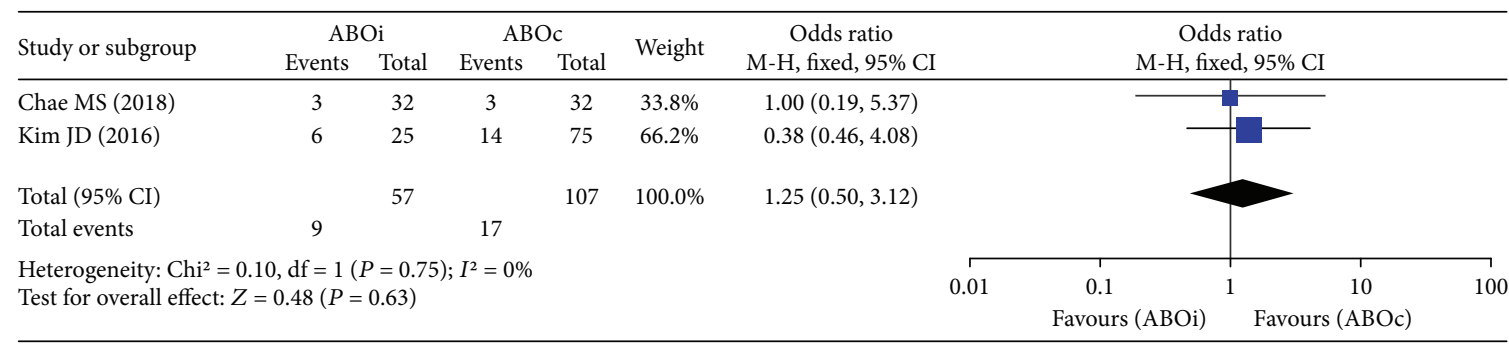

(a) Overall infections

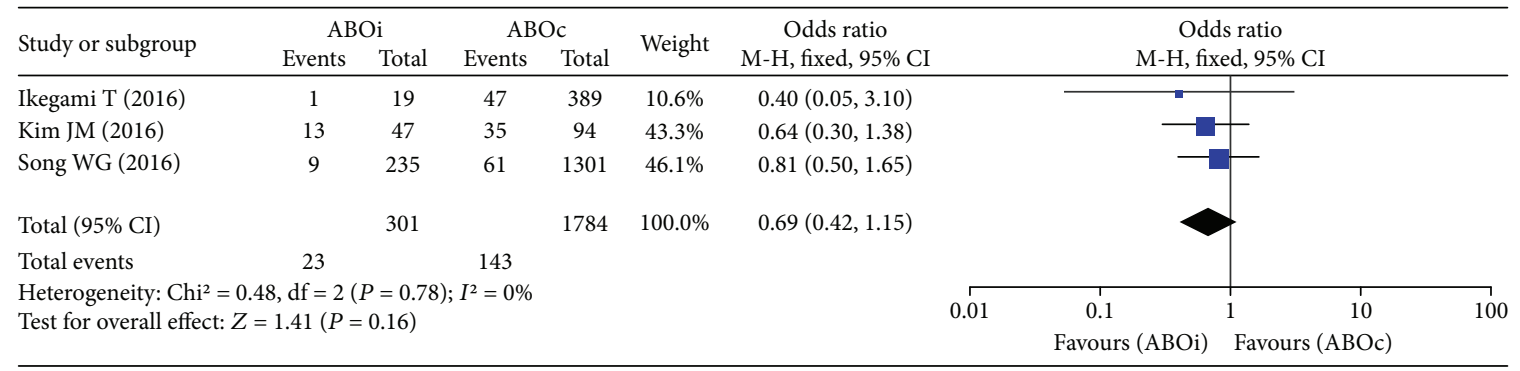

(b) Bacterial infections

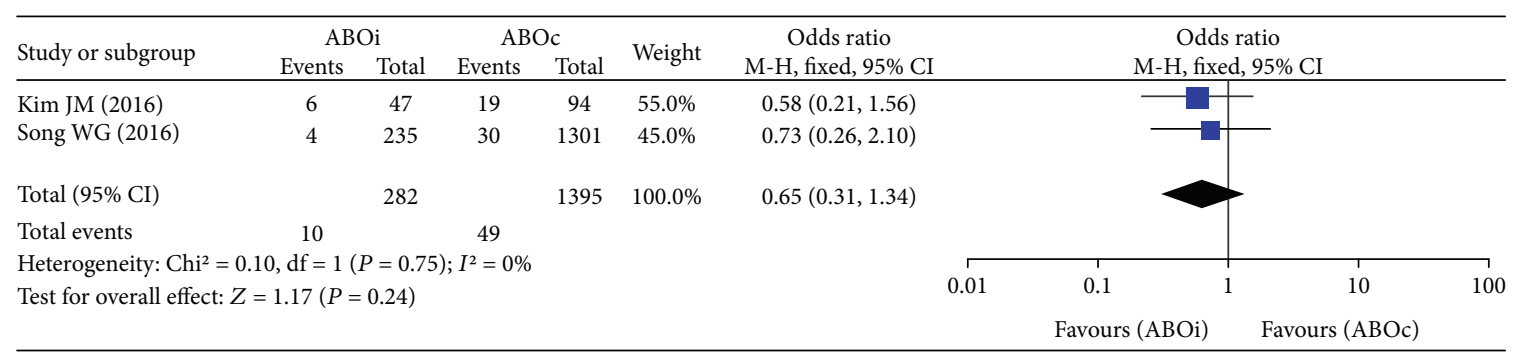

(c) Fungal infections

\begin{tabular}{|c|c|c|c|c|c|c|c|c|c|}
\hline \multirow{3}{*}{$\begin{array}{l}\text { Study or subgroup } \\
\text { Ikegami T (2016) }\end{array}$} & \multicolumn{2}{|c|}{ ABOi } & \multicolumn{2}{|c|}{$\mathrm{ABOc}$} & \multirow{2}{*}{ Weight } & \multirow{2}{*}{$\begin{array}{c}\text { Odds ratio } \\
\text { M-H, fixed, } 95 \% \mathrm{CI}\end{array}$} & \multirow{2}{*}{\multicolumn{2}{|c|}{$\begin{array}{c}\text { Odds ratio } \\
\text { M-H, fixed, } 95 \% \text { CI }\end{array}$}} & \\
\hline & \multirow{2}{*}{$\frac{\text { Events }}{10}$} & \multirow{2}{*}{$\frac{\text { Total }}{19}$} & \multirow{2}{*}{$\begin{array}{c}\text { Events } \\
89\end{array}$} & \multirow{2}{*}{$\frac{\text { Total }}{389}$} & & & & & \\
\hline & & & & & $17.5 \%$ & $3.75(1.48,9.50)$ & & 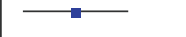 & \\
\hline Kim JM (2016) & 40 & 47 & 71 & 94 & $31.3 \%$ & $1.85(0.73,4.69)$ & & & \\
\hline Kim SH (2018) & 8 & 43 & 14 & 86 & $33.8 \%$ & $1.18(0.45,3.06)$ & & & \\
\hline Song WG (2016) & 3 & 235 & 13 & 1301 & $17.5 \%$ & $1.28(0.36,4.53)$ & & & \\
\hline Total $(95 \% \mathrm{CI})$ & & 344 & & 1870 & $100.0 \%$ & $1.85(1.13,3.03)$ & & & \\
\hline Total events & 61 & & 187 & & & & & & \\
\hline $\begin{array}{l}\text { Heterogeneity: Chi } \\
\text { Test for overall effec }\end{array}$ & $\begin{array}{l}\mathrm{df}=3( \\
2.46(P=\end{array}$ & $\begin{array}{l}=0.34 \\
0.01)\end{array}$ & $; I^{2}=11 \%$ & & & 0.01 & $\begin{array}{l}0.1 \\
\text { Favours (ABOi) }\end{array}$ & $\begin{array}{cc}10 \\
\text { Favours }(\mathrm{ABOc})\end{array}$ & 100 \\
\hline
\end{tabular}

(d) Cytomegalovirus (CMV) infection

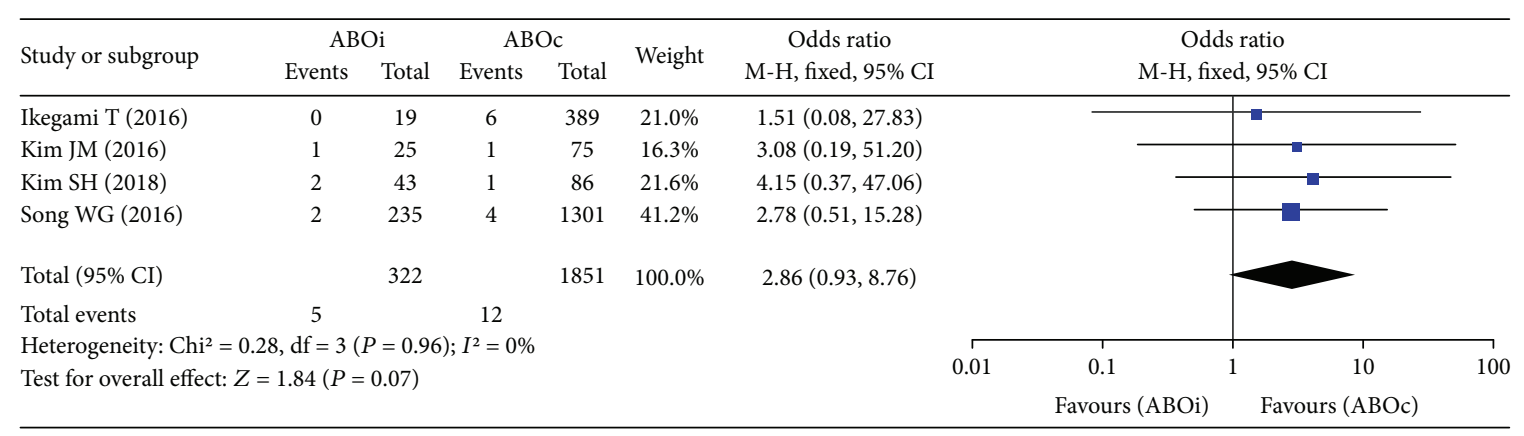

(e) Hepatic artery stenosis

Figure 3: Continued. 


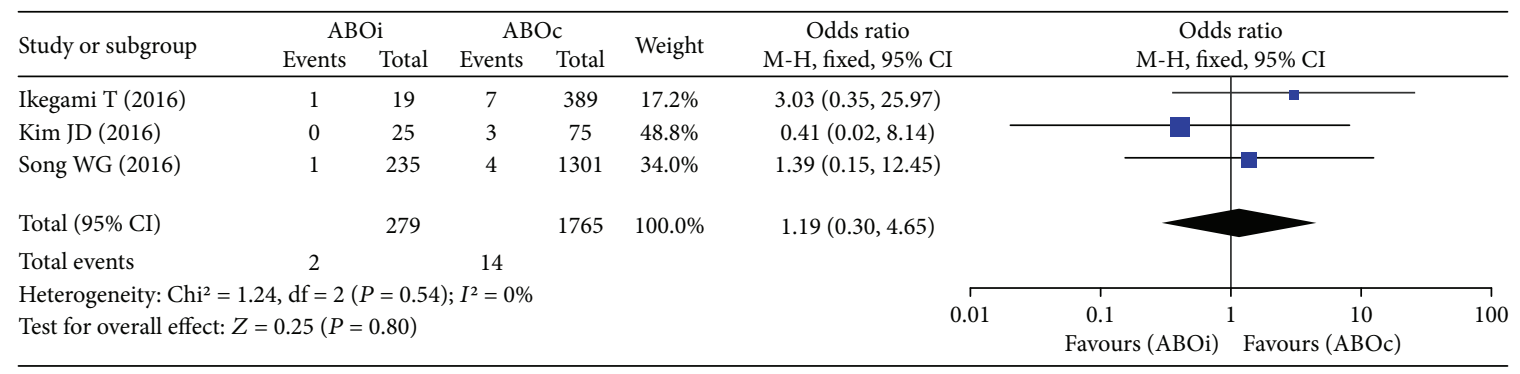

(f) Portal vein stenosis

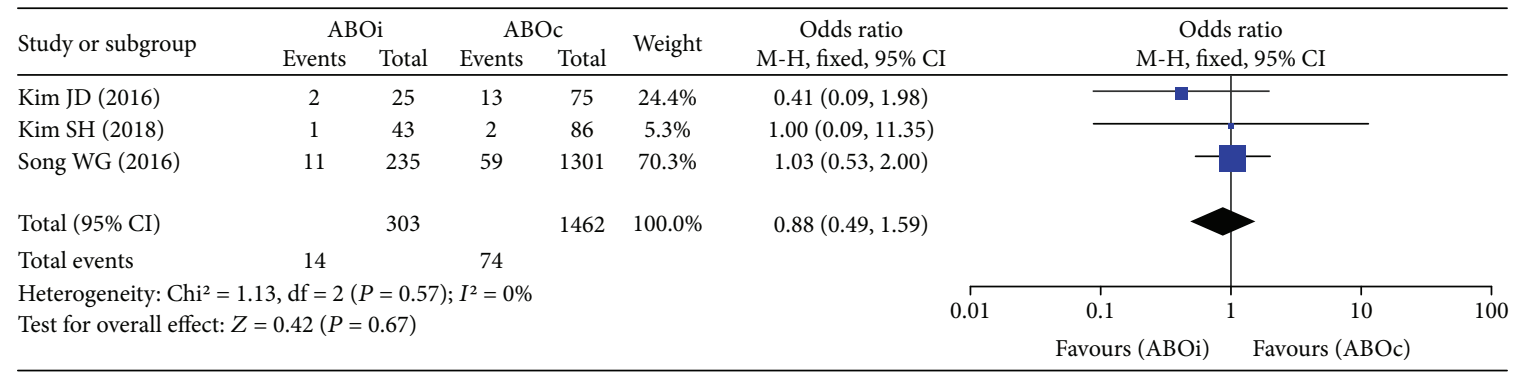

(g) Bleeding

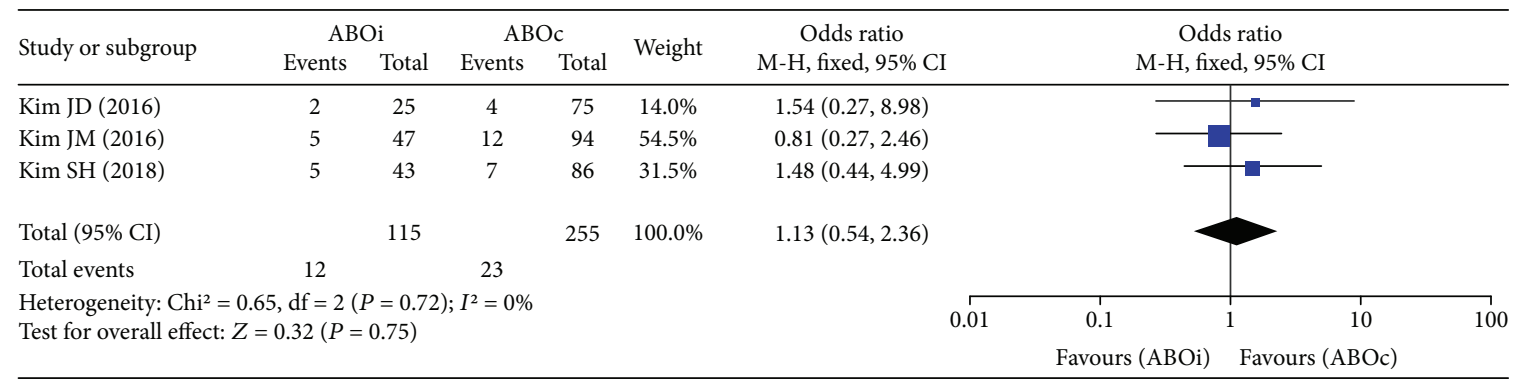

(h) Biliary leak

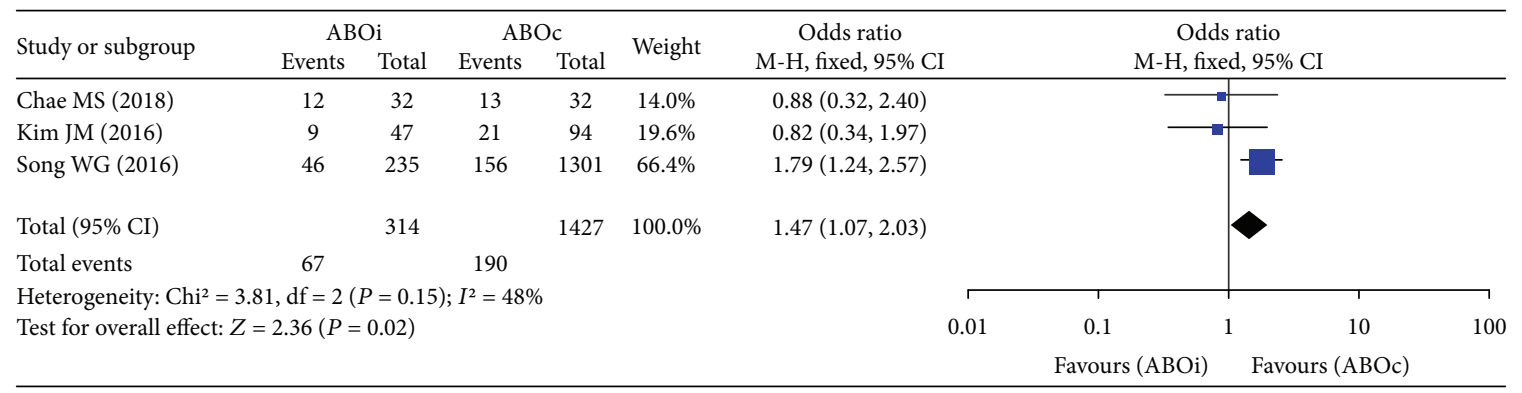

(i) Overall biliary complications

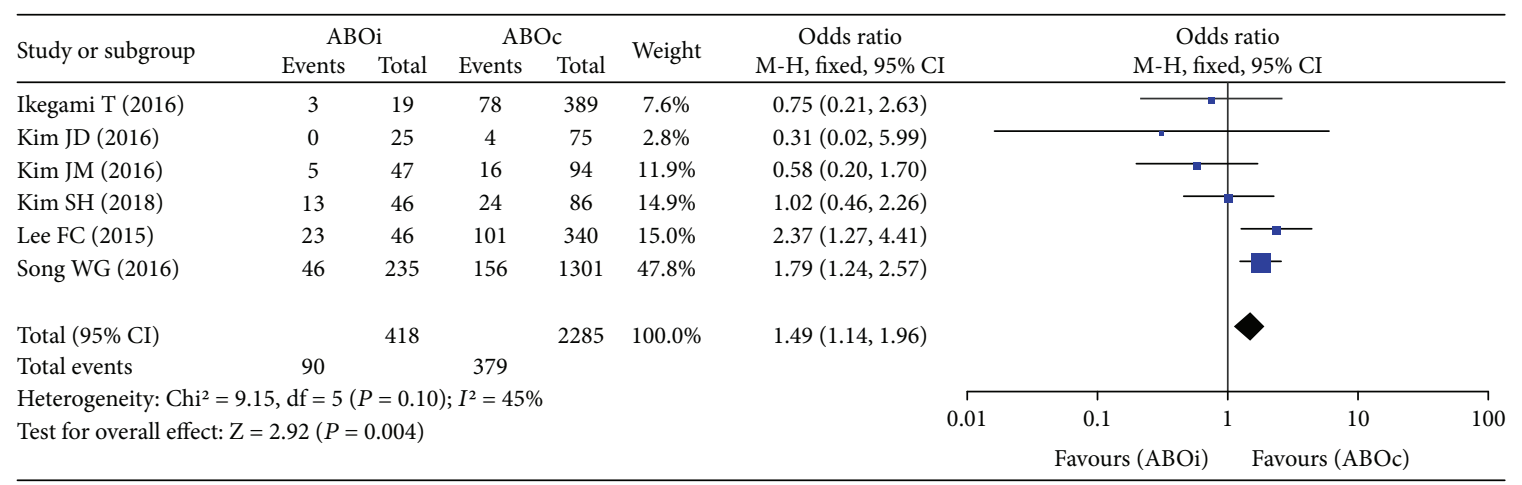

(j) Biliary stricture

FIgure 3: Continued. 


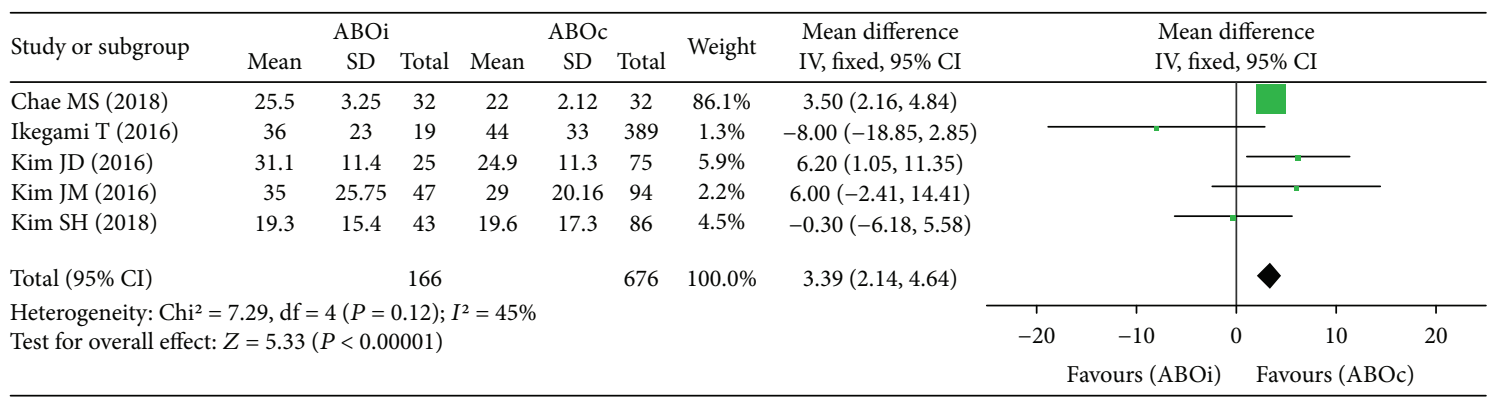

(k) Hospital stay

Figure 3: Forest plot of postoperative short-term outcomes: (a) overall infections, (b) bacterial infections, (c) fungal infections, (d) Cytomegalovirus (CMV) infection, (e) hepatic artery stenosis, (f) portal vein stenosis, (g) bleeding, (h) biliary leak, (i) overall biliary complications, (j) biliary stricture, and (k) hospital stay.

0.76 to $1.80, P=0.48$, Supplementary Figure 1(f)) OS, respectively. Furthermore, there were no data available to calculate OS stratified according to the Milan criteria.

To assess the outcome measurement of disease-free survival (DFS), data were classified according to 1-year and 3year DFS, respectively. There were no useful data to calculate 5-year DFS. A meta-analysis revealed that there was no significant difference between the $\mathrm{ABO}$ and $\mathrm{ABOc}$ groups for 1-year (OR: $1.26,95 \%$ CI: 0.76 to $2.09, P=0.37$, Supplementary Figure $1(\mathrm{~g})$ ) and 3-year (OR: $1.08,95 \% \mathrm{CI}: 0.74$ to 1.59 , $P=0.68$, Supplementary Figure 1(h)) DFS, respectively. Furthermore, classifying data according to the Milan criteria, a meta-analysis revealed that there was no significant difference between $\mathrm{ABO}$ and $\mathrm{ABOc}$ groups for 1-year (OR: $0.55,95 \% \mathrm{CI}: 0.27$ to $1.10, P=0.09$, Supplementary Figure 1(i)) and 3-year (OR: 0.22, 95\% CI: 0.01 to $3.50, P=0.28$, Supplementary Figure $1(\mathrm{j}))$ DFS beyond the Milan criteria. There were no useful data to calculate 5-year DFS beyond the Milan criteria. Moreover, there were also no useful data to calculate DFS within the Milan criteria.

\section{Discussion}

In spite of the colossal prospect of growing the donor pool through ABOi LDLT, the safety of ABOi ALDLT is debatable among the transplant community due to poor results in the recipients such as earlier graft loss, acute cellular rejection (ACR), antibody-mediated rejection (AMR), vascular complications, and biliary complications when compared to those of ABOc ALDLT [15-17]. The utilization of ABOi living donor is an alluring answer for growing the liver donor pool, and different novel procedures for the desensitization of $\mathrm{ABO}$ incompatibility have yielded promising outcomes $[11,12]$. However, earlier studies such as those not using rituximab in the desensitization protocol followed by ABOi LDLT showed inferior graft survival and patient survival compared to those of ABOc LDLT [15]. Nonetheless, the introduction of rituximab to the desensitization protocol has brought about significant improvements in the outcomes of ABOi LDLT [12, 19, 20].

ABOi LDLT in pediatric patients is considered safe and with acceptable results, probably because of their immature immune system $[11,13,14]$. Egawa et al. found that the 5 -year patient survival rate was significantly higher in infants than in adults ( $85 \%$ vs. 52\%) [17]. Similarly, several other studies found poor outcomes of ABOi LDLT in adults $[15,16]$. Thus, ABOi LDLT in adults seems to be controversial to many.

An effective desensitization protocol is the Achilles' heel of ABOi ALDLT. However, the standard desensitization protocol for ABOi ALDLT is yet to be implemented. Most of the centers use their own desensitization protocol with or without rituximab [15]. Thus, in the scenario of conflicting results from different studies, the most important attention regarding ABOi ALDLT is graft survival, posttransplant complications, and patient survival rate following ABOi ALDLT. A standard desensitization protocol should be designed by taking both the benefits and risks into consideration. Before the era of rituximab, the high incidence of early graft loss due to AMR was the major concern of ABOi LT [12, 35, 36]. However, the incidence of AMR decreased from $23.5 \%$ to $6.2 \%$ after the introduction of rituximab, as reported by a multicenter study from Japan [12].

To date, few systematic reviews and meta-analyses have been conducted comprehensively to analyze the short-term and long-term outcomes of ABOi and ABOc LT. However, an earlier meta-analysis was reported that was not specific to ABOi ALDLT after the introduction of rituximab in the desensitization protocol. Our meta-analysis includes nine relatively high-quality studies conducted between 2015 and 2018, all containing ALDLT using rituximab in the desensitization process for ABOi ALDLT, with a total of 3,858 patients $(\mathrm{ABO}=639$ and $\mathrm{ABOc}=3,219)$; thus, we believe it is the first study of its type.

In our meta-analysis, there was no significant difference between the ABOi and ABOc ALDLT groups in terms of 1-, 3-, and 5-year graft survival and overall survival. As reported by Egawa et al. [12], the significant reduction in the incidence of AMR after the introduction of rituximab might be the cause of the improvement in graft survival of ABOi ALDLT. Moreover, the largest single-center study by Song et al. [27] also reported similar outcomes in their study. Currently, there are no definitive answers as to why the overall survival of ABOi group did not differ from the ABOc group. Previously, some studies stated that the 


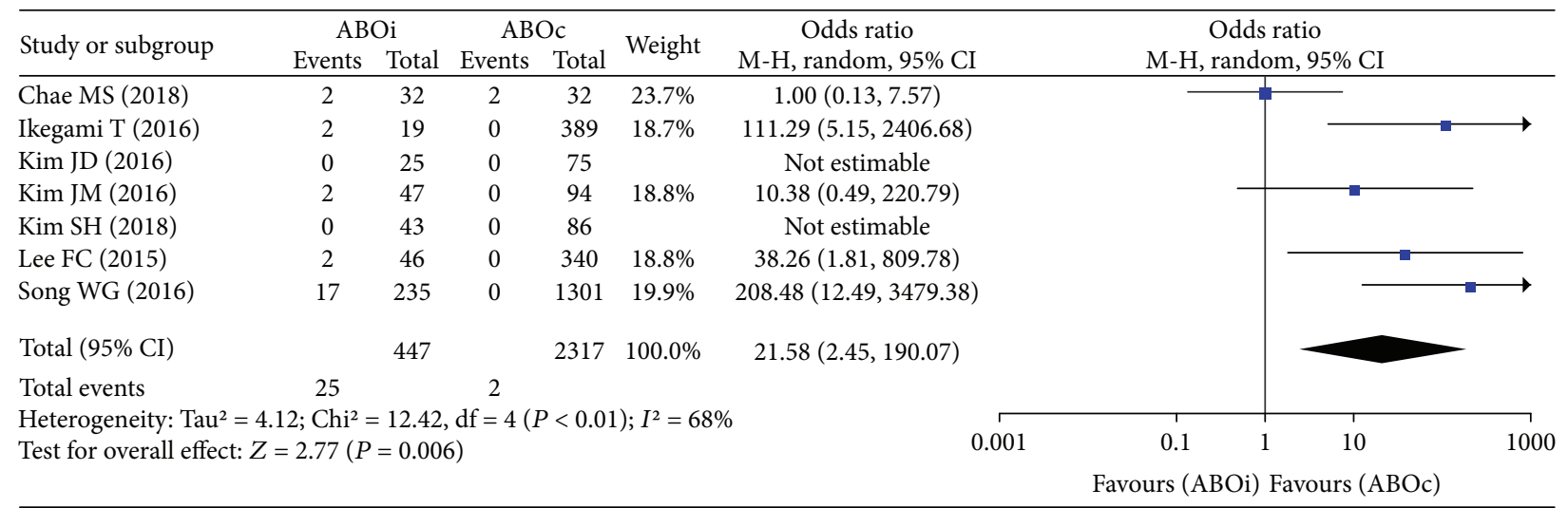

(a) Antibody-mediated rejection (AMR)

\begin{tabular}{|c|c|c|c|c|c|c|c|c|c|c|}
\hline \multirow{3}{*}{$\begin{array}{l}\text { Study or subgroup } \\
\text { Chae MS (2018) }\end{array}$} & \multicolumn{2}{|c|}{ ABOi } & \multicolumn{2}{|c|}{$\mathrm{ABOc}$} & \multirow{3}{*}{$\begin{array}{c}\text { Weight } \\
6.3 \%\end{array}$} & \multirow{3}{*}{$\begin{array}{c}\text { Odds ratio } \\
\text { M-H, fixed, 95\% CI } \\
1.30(0.31,5.35)\end{array}$} & \multirow{2}{*}{\multicolumn{3}{|c|}{$\begin{array}{c}\text { Odds ratio } \\
\mathrm{M}-\mathrm{H} \text {, fixed, } 95 \% \mathrm{CI}\end{array}$}} & \\
\hline & \multirow{2}{*}{ Events } & \multirow{2}{*}{$\begin{array}{c}\text { Total } \\
32\end{array}$} & \multirow{2}{*}{$\begin{array}{c}\text { Events } \\
4\end{array}$} & \multirow{2}{*}{$\begin{array}{c}\text { Total } \\
32\end{array}$} & & & & & & \\
\hline & & & & & & & & & & \\
\hline Ikegami T (2016) & 4 & 19 & 58 & 389 & $7.9 \%$ & $1.57(0.49,4.75)$ & & & & \\
\hline Kim JD (2016) & 0 & 25 & 1 & 75 & $1.4 \%$ & $0.97(0.04,24.67)$ & & & & \\
\hline Kim JM (2016) & 6 & 47 & 17 & 94 & $18.4 \%$ & $0.66(0.24,1.81)$ & & & & \\
\hline Kim SH (2018) & 0 & 43 & 1 & 86 & $1.9 \%$ & $0.66(0.03,16.42)$ & & & & \\
\hline Lee FC (2015) & 2 & 46 & 0 & 340 & $0.2 \%$ & $38.26(1.81,809.78)$ & & & & $\rightarrow$ \\
\hline Song (2016) & 19 & 235 & 122 & 1301 & $63.9 \%$ & $0.85(0.51,1.41)$ & & & & \\
\hline Total $(95 \%$ CI $)$ & & 447 & & 2317 & $100.0 \%$ & $0.98(0.67,1.43)$ & & & & \\
\hline Total events & 36 & & 203 & & & & & & & \\
\hline $\begin{array}{l}\text { Heterogeneity: Chi } \\
\text { Test for overall effe }\end{array}$ & $\begin{array}{l}\mathrm{f}=6(P \\
3(P=0\end{array}$ & $\begin{array}{l}0.30) \text {; } \\
0)\end{array}$ & $=17 \%$ & & & 0.01 & 0.1 & 1 & 10 & $\longrightarrow$ \\
\hline & & & & & & & Favo & & $\mathrm{ABOc})$ & \\
\hline
\end{tabular}

(b) Acute cellular rejection (ACR)

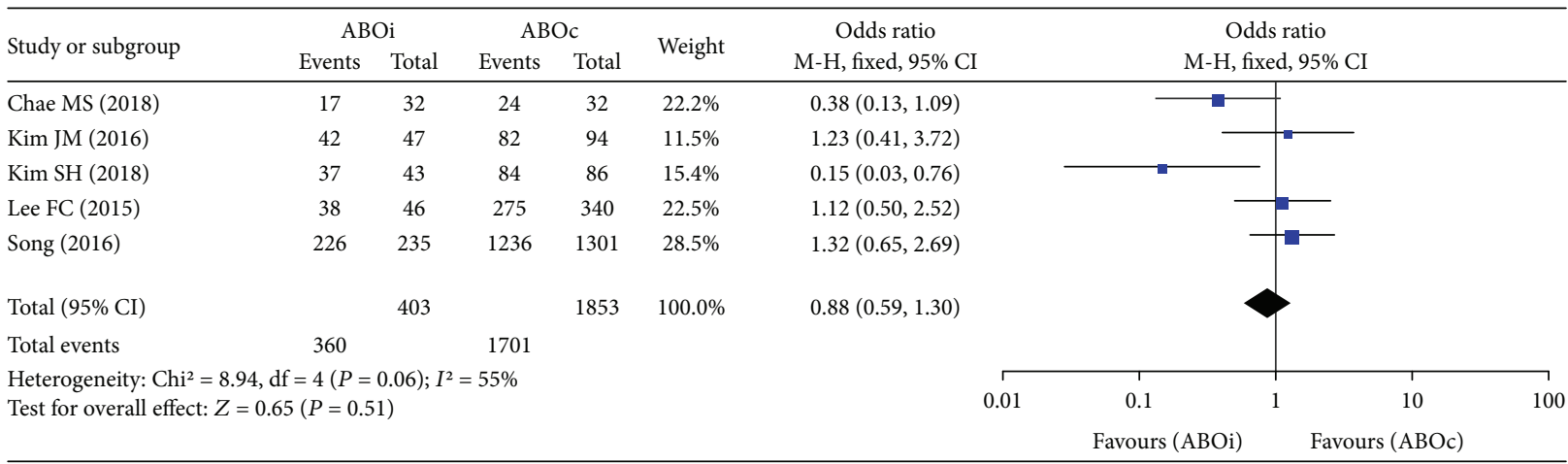

(c) 1 yr overall survival

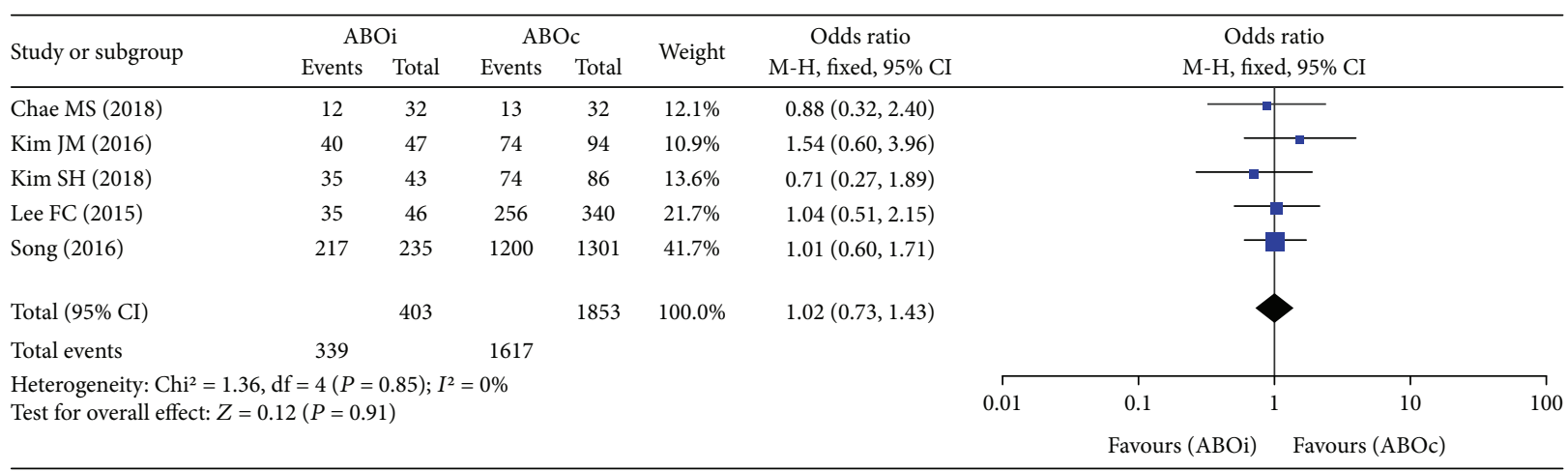

(d) 3 yr overall survival

Figure 4: Continued. 


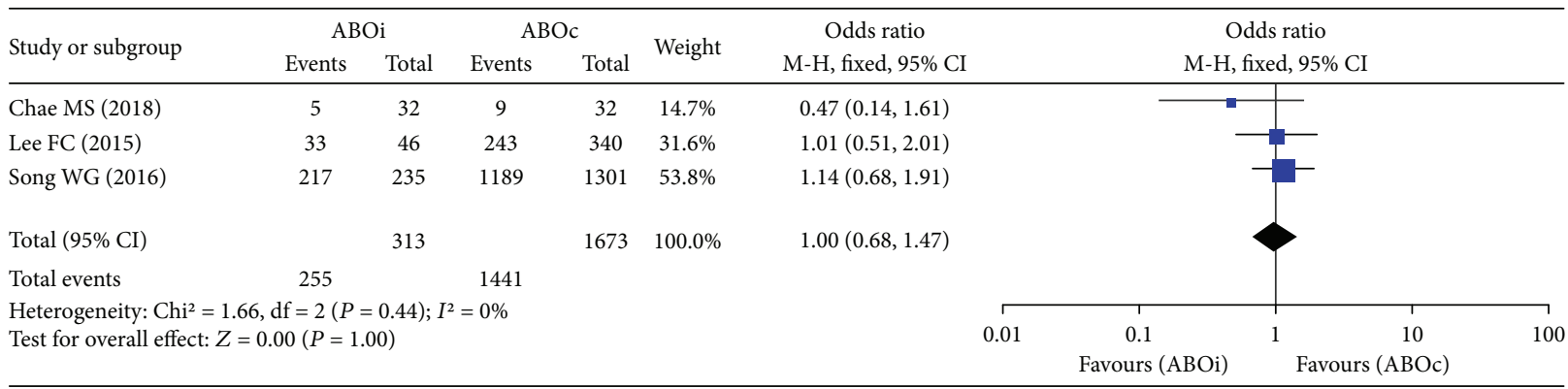

(e) 5 yr overall survival

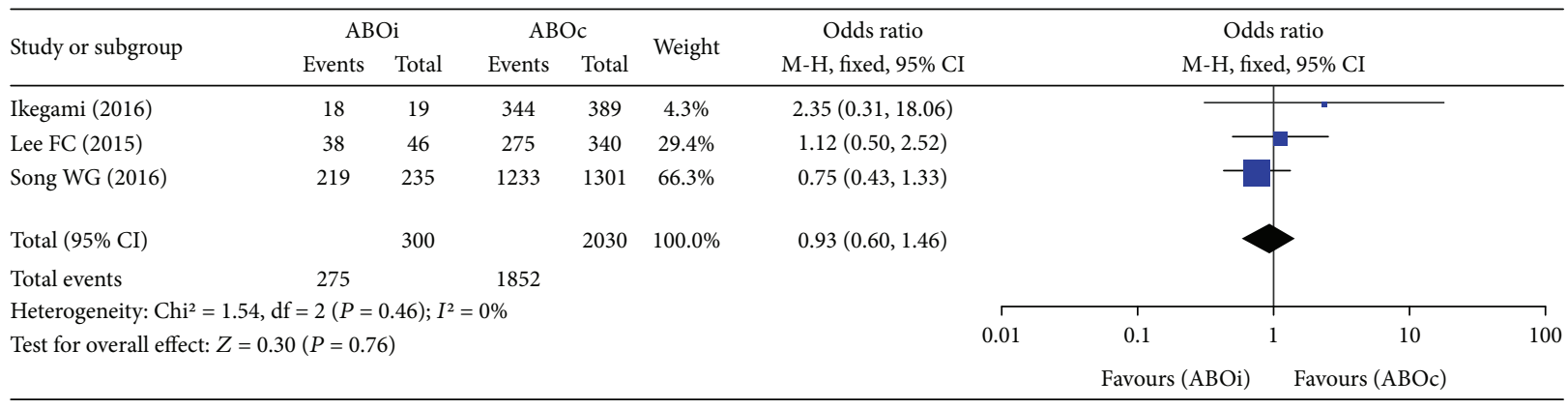

(f) 1 yr graft survival

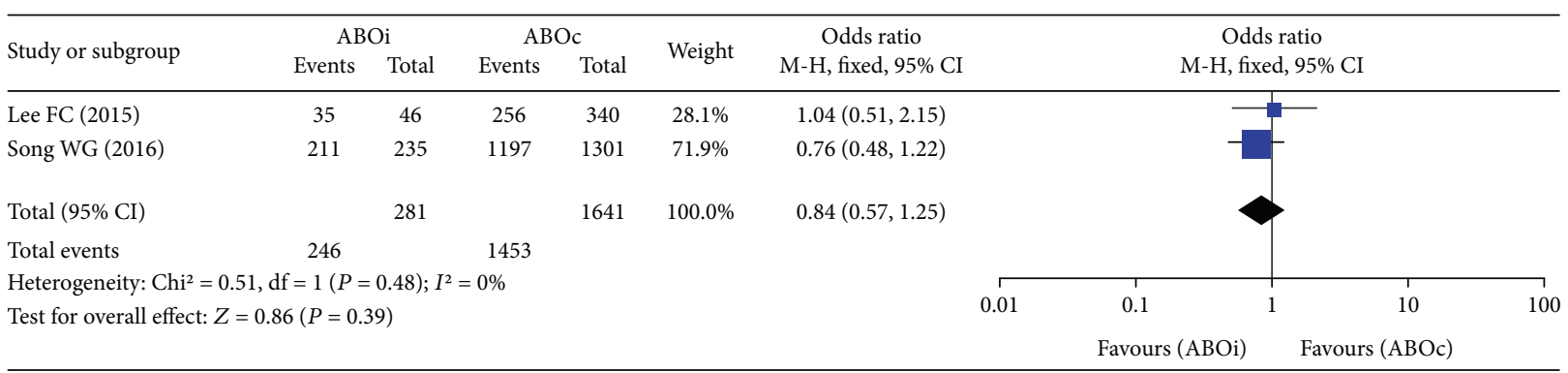

(g) 3 yr graft survival

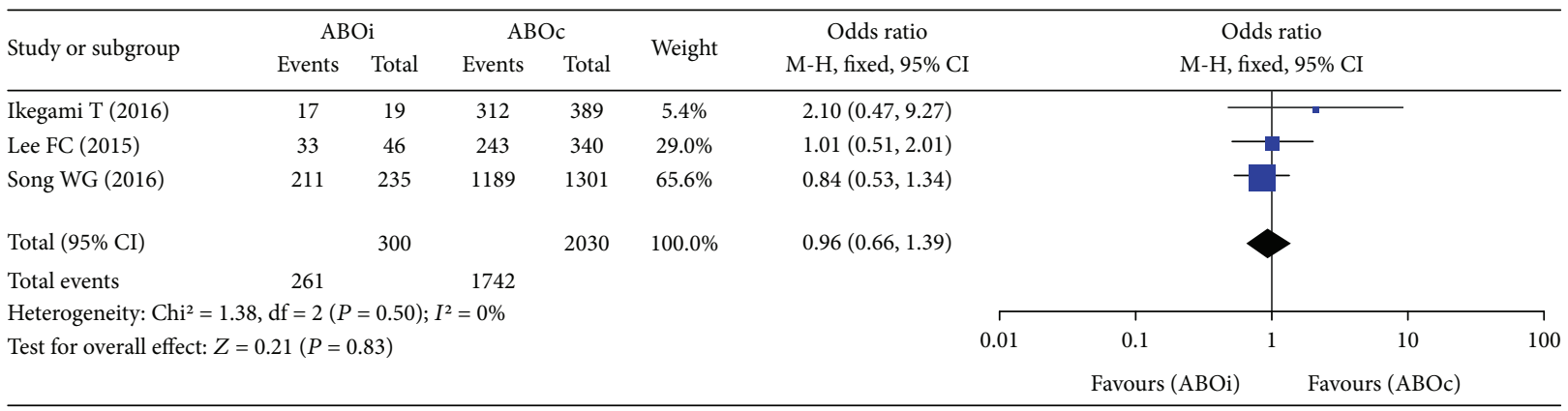

(h) 5 yr graft survival

FIGURE 4: Forest plot of postoperative long-term outcomes: (a) antibody-mediated rejection (AMR), (b) acute cellular rejection (ACR), (c) 1 yr overall survival, (d) $3 \mathrm{yr}$ overall survival, (e) $5 \mathrm{yr}$ overall survival, (f) $1 \mathrm{yr}$ graft survival, (g) $3 \mathrm{yr}$ graft survival, and (h) $5 \mathrm{yr}$ graft survival.

higher MELD score was the risk factor for patient survival after LT [17, 20, 27]. However, when we looked for a MELD score between the ABOi and ABOc groups, our meta-analysis did not find any significant difference between both groups.

The incidences of postoperative complications were comparable between both groups. However, ABOi ALDLT had higher incidences of CMV infection, AMR, overall biliary complications, and biliary stricture than adult ABOc ALDLT.
The possible cause of the higher incidence of CMV might be because of the immunocompromised state due to rituximab. Rituximab suppresses different stages of B cell differentiation leading to a rapid decrease in the peripheral $\mathrm{B}$ cell population within 48-72 hours, but which can last for several months[27, 37, 38]. Furthermore, repeated dosing of rituximab induces prolonged hypogammaglobulinemia which has a high risk for serious infectious complications [27, 38]. Likewise, in our meta-analysis two $[27,31]$ out of four studies reporting 
on CMV infection have used splenectomy in their desensitization protocol. Studies have shown that splenectomy is associated with a higher rate of serious infectious complications including CMV infection in LDLT [39]. Thus, a repeated dose of rituximab and inclusion of splenectomy in the desensitization protocol should be considered carefully. However, rituximab has additionally supplanted the need of splenectomy to prevent a posttransplant rebound increase of isohemagglutinins (IHs) [27, 40].

Despite the fact that after the introduction of rituximab to the desensitization protocol, the incidence of hepatic necrosis caused by AMR has disappeared, diffuse intrahepatic biliary stricture (DIHBS), which is a modest type of AMR, still remains to be the concern in ABOi ALDLT[19, 27]. Moreover, in the study by Song et al., DIHBS was reported to be solely in patients undergoing ABOi ALDLT [27]. The adequate reduction of $B$ cells and the elimination of serum IH titers are important steps concerning the prevention of $A M R[30]$. In instances of AMR, IHs initiate the immune response by binding to the graft vessels leading to the activation of the complement system and inflammation, which may further lead to hepatic artery thrombosis and necrosis of the liver $[36,41]$. Since ABO antigens are present on the bile duct epithelium, the activation of the immune response contributes to the increased incidence of uncompromising and continuous intrahepatic bile duct injury with ABOi LT [41]. As discussed earlier, rituximab, being an antiCD20 monoclonal antibody, can suppress the activated B cell population in circulation through antibody-dependent cellmediated cytotoxicity, direct antigen antibody reaction, and complement-dependent cytotoxicity; however, it is unable to suppress stem cells and plasma cells $[42,43]$. Interestingly, plasma B cells only get triggered after they encounter allografts after LT [30]. Moreover, it has also been reported that some B cells may rescue themselves preoperatively at the time of rituximab treatment and later can get activated after LT that produces antibodies [30]. Although rituximab may thoroughly control AMR over ABO barriers, it does not perform as such on the ground, so that it cannot annihilate plasma cells that are present on the epithelium of the bile ducts, thus leading to DIHBS and biliary stricture [31, 43]. This explains why ABOi ALDLT has a higher incidence of AMR, overall biliary complications, and biliary stricture. However, our meta-analysis showed that the ABOi group had a significantly shorter cold ischemia time than the ABOc group; the reason might be due to the concern of transplant surgeons to reduce the incidence of the ischemic type of biliary stricture. Nevertheless, this has not shown to improve the incidence of biliary stricture or overall biliary complications in the ABOi ALDLT group. Previously, some of the studies have outlined that the rise in posttransplant donorspecific antibody (DSA) titers is significantly associated with the incidence of AMR; therefore, an association of DSA should also be taken into consideration as the cause of AMR [44-46]. In our understanding, the most important key to avoid AMR in ABOi ALDLT is the inhibition of newly produced antibodies. TPE is a standard procedure to decrease DSA titers, yet the titer required to avoid AMR is not well characterized [47]. Furthermore, the dosing and timing of rituximab is also a concern regarding AMR [12, 27]. Egawa et al. [12] reported that consistent single doses of rituximab $\left(500 \mathrm{mg} / \mathrm{m}^{2}\right.$ or $\left.375 \mathrm{mg} / \mathrm{m}^{2}\right)$ had a lower frequency of AMR than a single low dose $\left(300 \mathrm{mg} / \mathrm{m}^{2}\right)$. In the context of comparison between TPE and rituximab, Kozaki et al. [48] found that rituximab was not sufficient for decreasing antibody titers after ABOi LDLT, and TPE remains to be a mainstay of treatment for such patients. On the other hand, Kim et al. [22] concluded that desensitization using rituximab and IVIG without TEP for ABOi LDLT was safe and effective in achieving sufficient desensitization with comparable outcomes.

Furthermore, a few case reports and series have been divulged in regard to the utilization of plasma cell depleting agents, such as bortezomib, in the treatment and prevention of AMR related with the anti-HLA antibody $[49,50]$. Bortezomib specifically prompts apoptosis among plasma cells, further diminishing isoagglutinin production [51]. However, further study is needed to prove the efficacy and safety of bortezomib combined with rituximab in the desensitization protocol for ABOi ALDLT.

Moreover, our review of studies suggested that there was no significant difference between the ABOi and ABOc groups for 1-year and 3-year OS and DFS for patients with HCC following ABOi ALDLT. However, the MELD score and the maximum tumor diameter were significantly lower in $\mathrm{ABO}$ ALDLT for patients with HCC, probably because of the careful patient selection for ABOi ALDLT. Both the studies reporting on the recurrence of $\mathrm{HCC}$ for ABOi ALDLT revealed that rituximab does not increase the risk of HCC recurrence $[25,26]$. Nevertheless, it has been found that overexposure to tacrolimus and basiliximab during the first year after LT increases the risk of HCC recurrence $[52,53]$.

Despite the high quality of the papers incorporated into this meta-analysis, there are various shortcomings concerning this meta-analysis. Firstly, there is a potential publication bias, because studies are less likely to outline negative findings. It could also be affected by the limited resources to identify unpublished trials. Secondly, only English-language studies were incorporated. Thus, the quality of outcomes was compromised to some extent, which is a typical reason for publication bias. Additionally, we could not identify two-arm studies comparing ABOi and ABOc ALDLT before the era of rituximab except for a few case reports or a onearm study; this would have been of great importance if the comparative results before and after the era of rituximab were established. Moreover, the studies included in this meta-analysis have used different desensitization protocols and immunotherapies after LT; thus, it was difficult to harmonize these different protocols to the results of the meta-analysis. However, this meta-analysis is still of great significance for comparing different outcomes between ABOi and ABOc ALDLT in the era of rituximab and may prove beneficial for the clinicians in choosing the appropriate strategy (Figure 5).

Our meta-analysis included the largest number of studies comparing $\mathrm{ABOi}$ and $\mathrm{ABOc}$ ALDLT and all those using rituximab prophylaxis for ABOi ALDLT. ABOi ALDLT showed comparable results with that of ABOc ALDLT. 


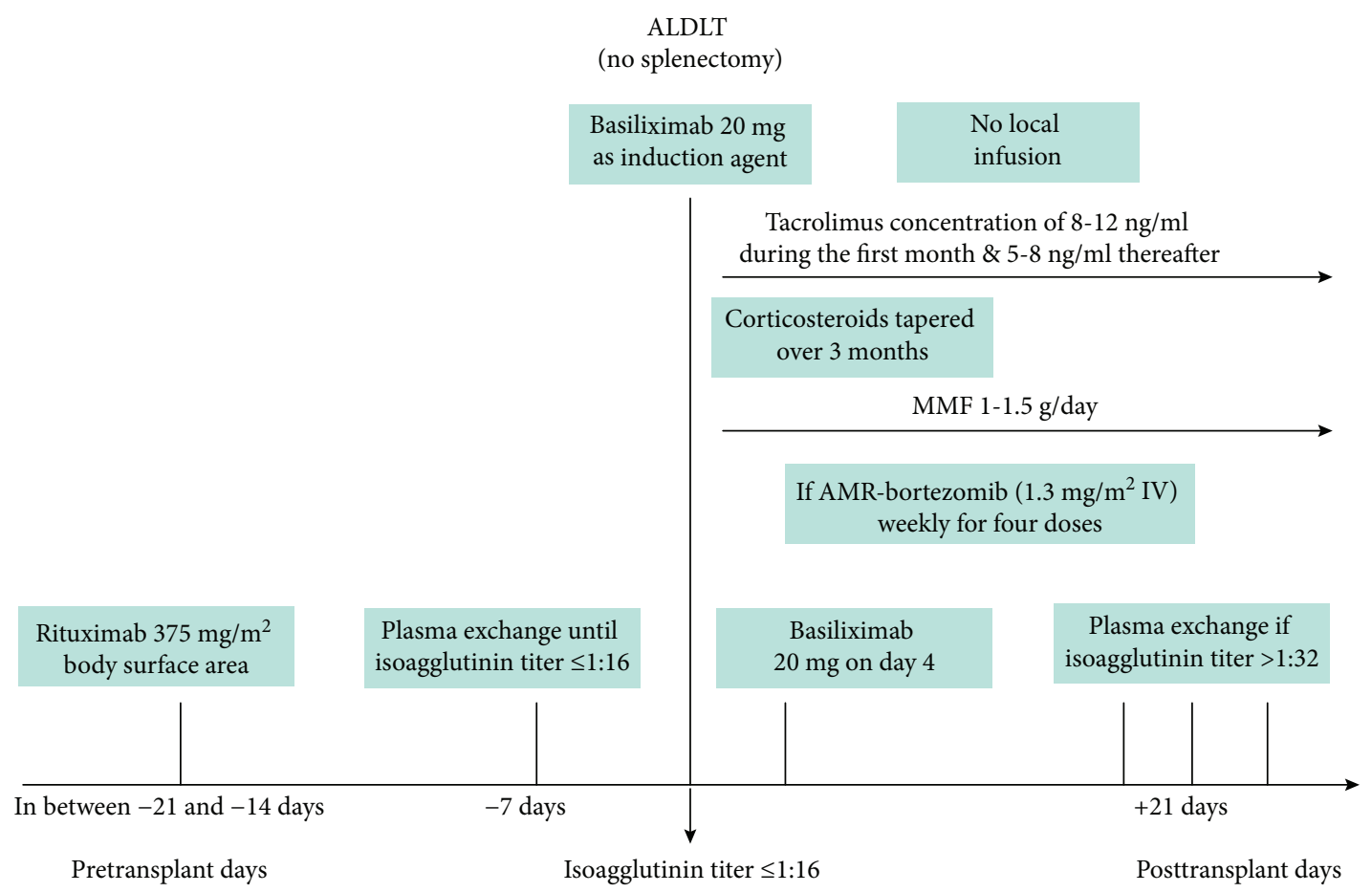

FIGURE 5: Suggested simplified desensitization protocol for ABO incompatible adult living donor liver transplantation. ALDLT—adult living donor liver transplantation; MMF_-mycophenolate mofetil; AMR—antibody-mediated rejection.

However, CMV infection, biliary stricture, and AMR remain the major concerns in the era of rituximab. Nevertheless, a clinical trial is required for the comparisons of patient outcomes with/without rituximab, dosing, and timing of rituximab in a large cohort; anyhow, it would be hard to withdraw rituximab prophylaxis when the current outcomes are so much promising in the era of rituximab. Thus, we suggest the need for an effective and standardized desensitization protocol in addition to rituximab in the future.

\section{Abbreviations}

ABOc: $\quad$ ABO compatible

ABOi: $\quad$ ABO incompatible

ACR: Acute cellular rejection

ALDLT: Adult living donor liver transplantation

AMR: Antibody-mediated rejection

CMV: Cytomegalovirus

ACR: Acute cellular rejection

HBV: Hepatitis B virus

HCC: Hepatocellular carcinoma

$\mathrm{HCV}$ : Hepatitis $\mathrm{C}$ virus

IHs: Isohemagglutinins

LDLT: Living donor liver transplantation

LT: $\quad$ Liver transplantation

MELD: Model for end-stage liver disease

AFP: alpha-Fetoprotein

TPE: Total plasma exchange

IVIG: Intravenous immunoglobulin G

DFS: Disease-free survival

OS: Overall survival.

\section{Disclosure}

This manuscript was presented as a poster in the 2nd International Advanced Liver and Pancreas Surgery Symposium, Seoul, Korea.

\section{Conflicts of Interest}

The authors declare no competing interests.

\section{Acknowledgments}

This work was supported by grants from the 973 program (No. 2014CB542101), the National Natural Science Foundation of China (No. 81472212), the Key Program of Medical Scientific Research Foundation of Zhejiang Province, China (No. WKJ-ZJ-1410), the Key Program of Administration of Traditional Chinese Medicine of Zhejiang Province, China (No. 2014ZZ00), and the Zhejiang Provincial Program for the Cultivation of High-level Innovative Health Talents.

\section{Supplementary Materials}

Supplementary Table 1 Newcastle-Ottawa quality assessment scale. Supplementary Figure 1: forest plot of HCC patients. (a) AFP level, (b) MELD score for HCC patients, (c) maximum tumor diameter, (d) number of tumors, (e) 1-year overall survival for HCC patients, (f) 3-year overall survival for HCC patients, (g) 1-year disease-free survival, (h) 3-year disease-free survival, (i) 1-year disease-free survival beyond Milan criteria, and (j) 3-year disease-free survival beyond Milan criteria. (Supplementary Materials) 


\section{References}

[1] J. Bruix, M. Sherman, and American Association for the Study of Liver Diseases, "Management of hepatocellular carcinoma: an update," Hepatology, vol. 53, no. 3, pp. 1020-1022, 2011.

[2] A. Forner, J. M. Llovet, and J. Bruix, "Hepatocellular carcinoma," The Lancet, vol. 379, no. 9822, pp. 1245-1255, 2012.

[3] P. G. Northup, N. M. Intagliata, N. L. Shah, S. J. Pelletier, C. L. Berg, and C. K. Argo, "Excess mortality on the liver transplant waiting list: unintended policy consequences and Model for End-Stage Liver Disease (MELD) inflation," Hepatology, vol. 61, no. 1, pp. 285-291, 2015.

[4] H. Yeh, E. Smoot, D. A. Schoenfeld, and J. F. Markmann, "Geographic inequity in access to livers for transplantation," Transplantation, vol. 91, no. 4, pp. 479-486, 2011.

[5] G. Sapisochin and J. Bruix, "Liver transplantation for hepatocellular carcinoma: outcomes and novel surgical approaches," Nature reviews Gastroenterology \& Hepatology, vol. 14, no. 4, pp. 203-217, 2017.

[6] R. A. Fisher, "Living donor liver transplantation: eliminating the wait for death in end-stage liver disease?," Nature reviews Gastroenterology \& Hepatology, vol. 14, no. 6, pp. 373-382, 2017.

[7] M. Stegall, "ABO-incompatible liver transplant: is it justifiable?," Liver Transplantation, vol. 9, no. 1, p. 31, 2003.

[8] D. W. Hanto, A. H. Fecteau, M. H. Alonso, J. F. Valente, and J. F. Whiting, “ABO-incompatible liver transplantation with no immunological graft losses using total plasma exchange, splenectomy, and quadruple immunosuppression: evidence for accommodation," Liver Transplantation, vol. 9, no. 1, pp. 22-30, 2003.

[9] R. Watson, T. Kozlowski, V. Nickeleit et al., "Isolated donor specific alloantibody-mediated rejection after ABO compatible liver transplantation," American Journal of Transplantation, vol. 6, no. 12, pp. 3022-3029, 2006.

[10] R. B. Colvin, "C4d in liver allografts: a sign of antibodymediated rejection?," American Journal of Transplantation, vol. 6, no. 3, pp. 447-448, 2006.

[11] S. Rummler, A. Bauschke, E. Bärthel et al., "Current techniques for AB0-incompatible living donor liver transplantation," World Journal of Transplantation, vol. 6, no. 3, pp. 548-555, 2016.

[12] H. Egawa, S. Teramukai, H. Haga et al., "Impact of rituximab desensitization on blood-type-incompatible adult living donor liver transplantation: a Japanese multicenter study," American Journal of Transplantation, vol. 14, no. 1, pp. 102-114, 2014.

[13] T. Yandza, T. Lambert, F. Alvarez et al., "Outcome of ABOincompatible liver transplantation in children with no specific alloantibodies at the time of transplantation," Transplantation, vol. 58, no. 1, pp. 46-50, 1994.

[14] T. H. Renard and W. S. Andrews, "An approach to ABOincompatible liver transplantation in children," Transplantation, vol. 53, no. 1, pp. 116-120, 1992.

[15] E. C. Lee, S. H. Kim, and S.-J. Park, "Outcomes after liver transplantation in accordance with $\mathrm{ABO}$ compatibility: a systematic review and meta-analysis," World Journal of Gastroenterology, vol. 23, no. 35, pp. 6516-6533, 2017.

[16] J. Wu, S. Ye, X. Xu, H. Xie, L. Zhou, and S. Zheng, "Recipient outcomes after $\mathrm{ABO}$-incompatible liver transplantation: a systematic review and meta-analysis," PLOS ONE, vol. 6, no. 1, article e16521, 2011.
[17] H. Egawa, S. Teramukai, H. Haga, M. Tanabe, M. Fukushima, and M. Shimazu, "Present status of ABO-incompatible living donor liver transplantation in Japan," Hepatology, vol. 47, no. 1, pp. 143-152, 2008.

[18] S. Miyagi, N. Kawagishi, S. Sekiguchi et al., "The relationship between recurrences and immunosuppression on living donor liver transplantation for hepatocellular carcinoma," Transplantation Proceedings, vol. 44, no. 3, pp. 797-801, 2012.

[19] G. W. Song, S. G. Lee, S. Hwang et al., "Biliary stricture is the only concern in ABO-incompatible adult living donor liver transplantation in the rituximab era," Journal of Hepatology, vol. 61, no. 3, pp. 575-582, 2014.

[20] J. M. Kim, C. H. D. Kwon, J.-W. Joh et al., “ABO-incompatible living donor liver transplantation is suitable in patients without ABO-matched donor," Journal of Hepatology, vol. 59, no. 6, pp. 1215-1222, 2013.

[21] H. Egawa, K. Ohmori, H. Haga et al., "B-cell surface marker analysis for improvement of rituximab prophylaxis in $\mathrm{ABO}$ incompatible adult living donor liver transplantation," Liver Transplantation, vol. 13, no. 4, pp. 579-588, 2007.

[22] S. H. Kim, E. C. Lee, J. R. Shim, and S. J. Park, “A simplified protocol using rituximab and immunoglobulin for $\mathrm{ABO}$ incompatible low-titre living donor liver transplantation," Liver International, vol. 38, no. 5, pp. 932-939, 2018.

[23] I. Monteiro, L. M. McLoughlin, A. Fisher, A. N. de la Torre, and B. Koneru, "Rituximab with plasmapheresis and splenectomy in ABO-incompatible liver transplantation," Transplantation, vol. 76, no. 11, pp. 1648-1649, 2003.

[24] M. S. Chae, N. Lee, H. J. Choi et al., "Comparison of liver graft regeneration between $\mathrm{ABO}$-compatible and $\mathrm{ABO}$ incompatible living donor liver transplantation: a propensity score matching analysis," Annals of Transplantation, vol. 23, pp. 507-519, 2018.

[25] J. M. Kim, C. H. D. Kwon, J. W. Joh et al., “ABO-incompatible living donor liver transplantation with rituximab and total plasma exchange does not increase hepatocellular carcinoma recurrence," Transplantation, vol. 102, no. 10, pp. 16951701, 2018.

[26] Y. I. Yoon, G. W. Song, S. G. Lee et al., "Outcome of ABOincompatible adult living-donor liver transplantation for patients with hepatocellular carcinoma," Journal of Hepatology, vol. 68, no. 6, pp. 1153-1162, 2018.

[27] G.-. W. Song, S.-. G. Lee, S. Hwang et al., “ABO-incompatible adult living donor liver transplantation under the desensitization protocol with rituximab," American Journal of Transplantation, vol. 16, no. 1, pp. 157-170, 2016.

[28] J. M. Kim, C. H. D. Kwon, J.-W. Joh et al., "Case-matched comparison of $\mathrm{ABO}$-incompatible and $\mathrm{ABO}$-compatible living donor liver transplantation," British Journal of Surgery, vol. 103, no. 3, pp. 276-283, 2016.

[29] J. D. Kim, D. L. Choi, S. G. Kim, and A. J. Lee, "Single-center experience of $\mathrm{ABO}$-incompatible living-donor liver transplantation with a new simplified intravenous immunoglobulin protocol: a propensity score-matching analysis," Transplantation Proceedings, vol. 48, no. 4, pp. 1134-1138, 2016.

[30] C. F. Lee, C. H. Cheng, Y. C. Wang et al., "Adult living donor liver transplantation across $\mathrm{ABO}$-incompatibility," Medicine, vol. 94, no. 42, article e1796, 2015.

[31] T. Ikegami, T. Yoshizumi, Y. Soejima, H. Uchiyama, K. Shirabe, and Y. Maehara, "Feasible usage of ABO 
incompatible grafts in living donor liver transplantation," Hepatobiliary Surgery and Nutrition, vol. 5, no. 2, pp. 91-97, 2016.

[32] D. Moher, A. Liberati, J. Tetzlaff, D. G. Altman, and the PRISMA Group, "Preferred reporting items for systematic reviews and meta-analyses: the PRISMA statement," Annals of Internal Medicine, vol. 151, no. 4, pp. 264-269, 2009.

[33] C. K.-L. Lo, D. Mertz, and M. Loeb, "Newcastle-Ottawa Scale: comparing reviewers' to authors' assessments," BMC Medical Research Methodology, vol. 14, no. 1, p. 45, 2014.

[34] J. P. Higgins, S. G. Thompson, J. J. Deeks, and D. G. Altman, "Measuring inconsistency in meta-analyses," BMJ, vol. 327, no. 7414, pp. 557-560, 2003.

[35] J. Gugenheim, D. Samuel, H. Bismuth, and M. Reynes, "Liver transplantation across ABO blood group barriers," The Lancet, vol. 336, no. 8714, pp. 519-523, 1990.

[36] A. J. Demetris, R. Jaffe, A. Tzakis et al.et al., “Antibody-mediated rejection of human orthotopic liver allografts: a study of liver transplantation across ABO blood group barriers," The American Journal of Pathology, vol. 132, no. 3, pp. 489-502, 1988.

[37] D. Toki, H. Ishida, S. Horita, K. Setoguchi, Y. Yamaguchi, and $\mathrm{K}$. Tanabe, "Impact of low-dose rituximab on splenic B cells in ABO-incompatible renal transplant recipients," Transplant, vol. 22, no. 4, pp. 447-454, 2009.

[38] H. Genberg, A. Hansson, A. Wernerson, L. Wennberg, and G. Tyden, "Pharmacodynamics of rituximab in kidney allotransplantation," American Journal of Transplantation, vol. 6, no. 10, pp. 2418-2428, 2006.

[39] A. Badawy, Y. Hamaguchi, S. Satoru, T. Kaido, H. Okajima, and S. Uemoto, "Evaluation of safety of concomitant splenectomy in living donor liver transplantation: a retrospective study," Transplant International, vol. 30, no. 9, pp. 914-923, 2017.

[40] S. D. Lee, S. H. Kim, S. Y. Kong, Y. K. Kim, S. A. Lee, and S. J. Park, "ABO-incompatible living donor liver transplantation without graft local infusion and splenectomy," $H P B$, vol. 16, no. 9, pp. 807-813, 2014.

[41] L. Sanchez-Urdazpal, K. P. Batts, G. J. Gores et al., "Increased bile duct complications in liver transplantation across the ABO barrier," Annals of Surgery, vol. 218, no. 2, pp. 152-158, 1993.

[42] P. Boross and J. H. Leusen, "Mechanisms of action of CD20 antibodies," American Journal of Cancer Research, vol. 2, no. 6, pp. 676-690, 2012.

[43] G. J. Weiner, "Rituximab: mechanism of action," Seminars in Hematology, vol. 47, no. 2, pp. 115-123, 2010.

[44] M. Kasahara, T. Kiuchi, K. Takakura et al., "Postoperative flow cytometry crossmatch in living donor liver transplantation: clinical significance of humoral immunity in acute rejection," Transplantation, vol. 67, no. 4, pp. 568-575, 1999.

[45] L. J. Wozniak, M. J. Hickey, R. S. Venick et al., "Donor-specific HLA antibodies are associated with late allograft dysfunction after pediatric liver transplantation," Transplantation, vol. 99, no. 7, pp. 1416-1422, 2015.

[46] J. G. O’Leary, H. Kaneku, B. M. Susskind et al., "High mean fluorescence intensity donor-specific anti-HLA antibodies associated with chronic rejection postliver transplant," American Journal of Transplantation, vol. 11, no. 9, pp. 1868-1876, 2011.
[47] M. Honda, Y. Sugawara, M. Kadohisa et al., "Long-term outcomes of ABO-incompatible pediatric living donor liver transplantation," Transplantation, vol. 102, no. 10, pp. 1702 1709, 2018.

[48] K. Kozaki, H. Egawa, M. Ueda et al., "The role of apheresis therapy for $\mathrm{ABO}$ incompatible living donor liver transplantation: the Kyoto University experience," Therapeutic Apheresis and Dialysis, vol. 10, no. 5, pp. 441-448, 2006.

[49] S. M. Flechner, R. Fatica, M. Askar et al., "The role of proteasome inhibition with bortezomib in the treatment of antibody-mediated rejection after kidney-only or kidneycombined organ transplantation," Transplantation, vol. 90, no. 12, pp. 1486-1492, 2010.

[50] F. Paterno, M. Shiller, G. Tillery et al., "Bortezomib for outcomes of ABO-incompatible pediatric living donor liver transplantation," American Journal of Transplantation, vol. 12, no. 9, pp. 2526-2531, 2012.

[51] D. K. Perry, J. M. Burns, H. S. Pollinger et al., "Proteasome inhibition causes apoptosis of normal human plasma cells preventing alloantibody production," American Journal of Transplantation, vol. 9, no. 1, pp. 201-209, 2009.

[52] J. R. Parfitt, P. Marotta, M. AlGhamdi et al., "Recurrent hepatocellular carcinoma after transplantation: use of a pathological score on explanted livers to predict recurrence," Liver Transplantation, vol. 13, no. 4, pp. 543-551, 2007.

[53] J.-Y. Lee, Y. H. Kim, N.-J. Yi et al., "Impact of immunosuppressant therapy on early recurrence of hepatocellular carcinoma after liver transplantation," Clinical and Molecular Hepatology, vol. 20, no. 2, pp. 192-203, 2014. 


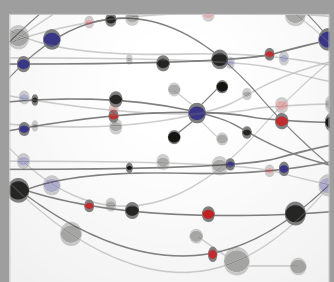

The Scientific World Journal
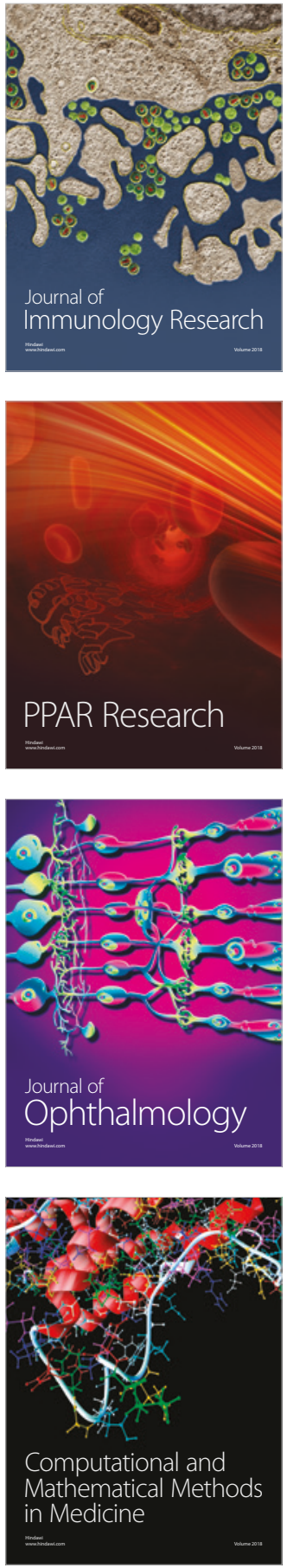

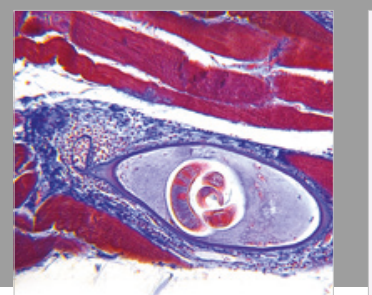

Gastroenterology Research and Practice

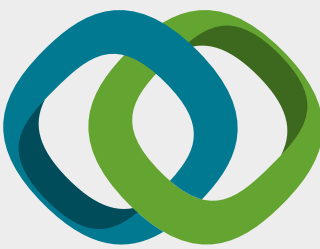

\section{Hindawi}

Submit your manuscripts at

www.hindawi.com
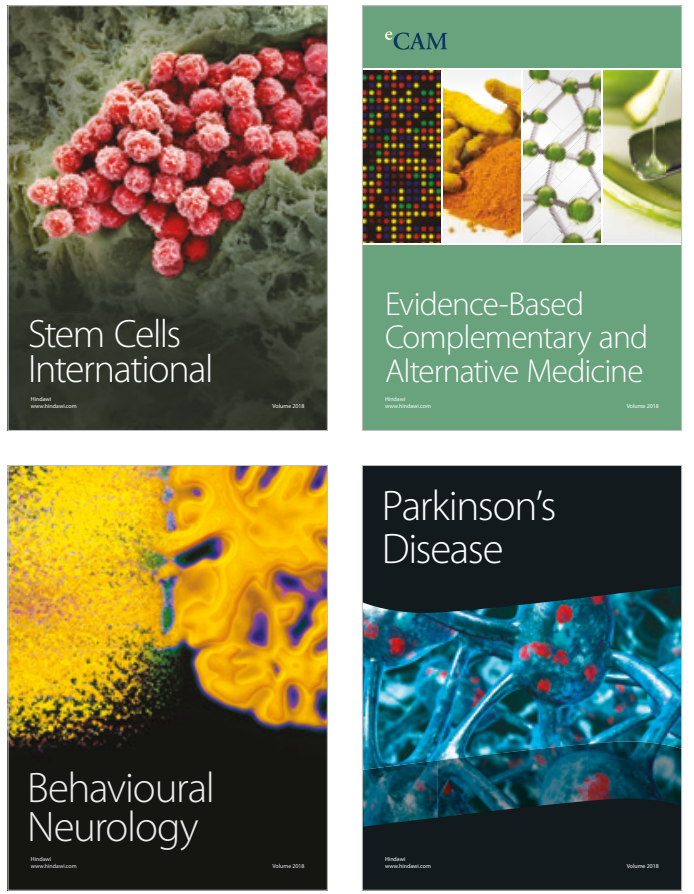

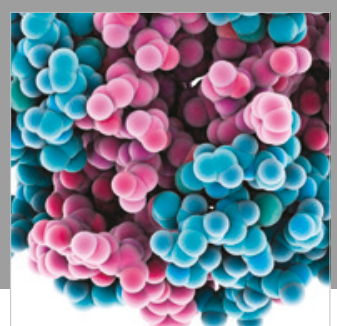

ournal of

Diabetes Research

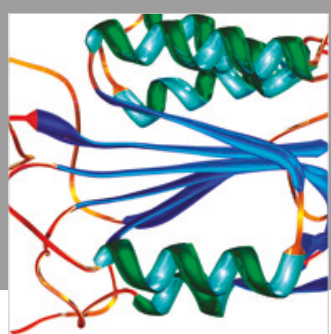

Disease Markers
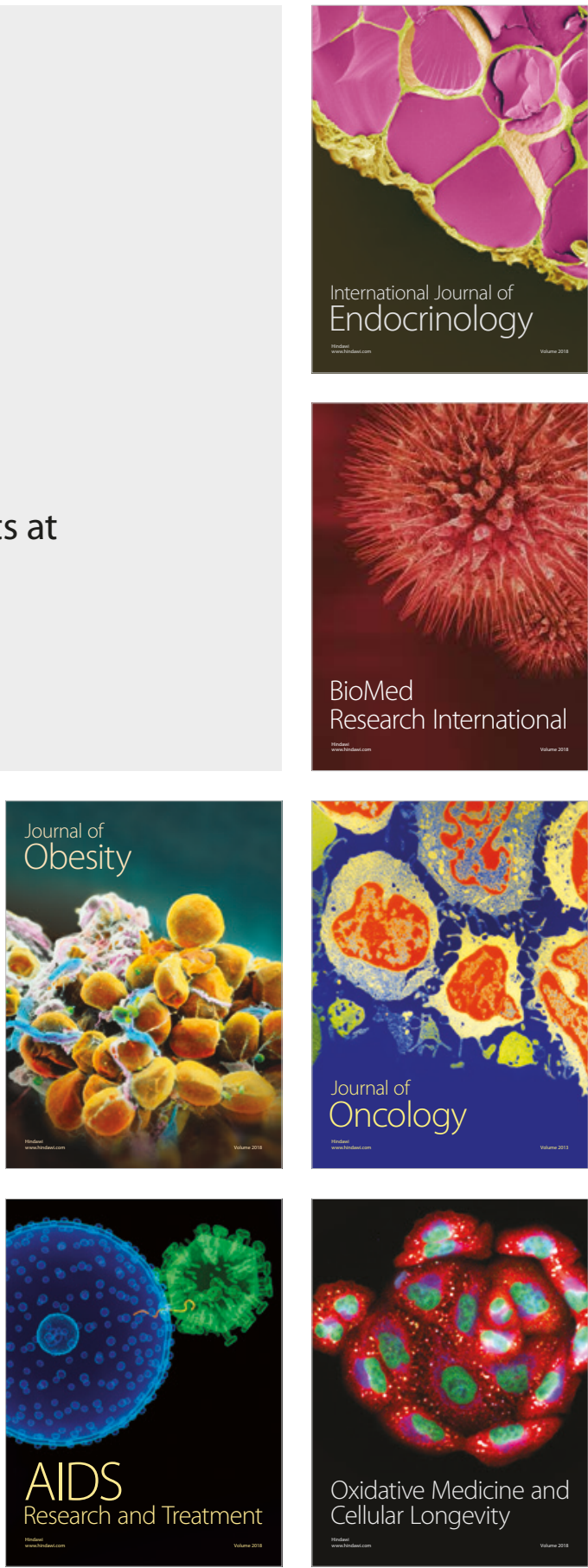